Ciencia, tecnología e innovación en México: un análisis de la política pública

Claudia Díaz Pérez y Moisés Alejandro Alarcón Osuna
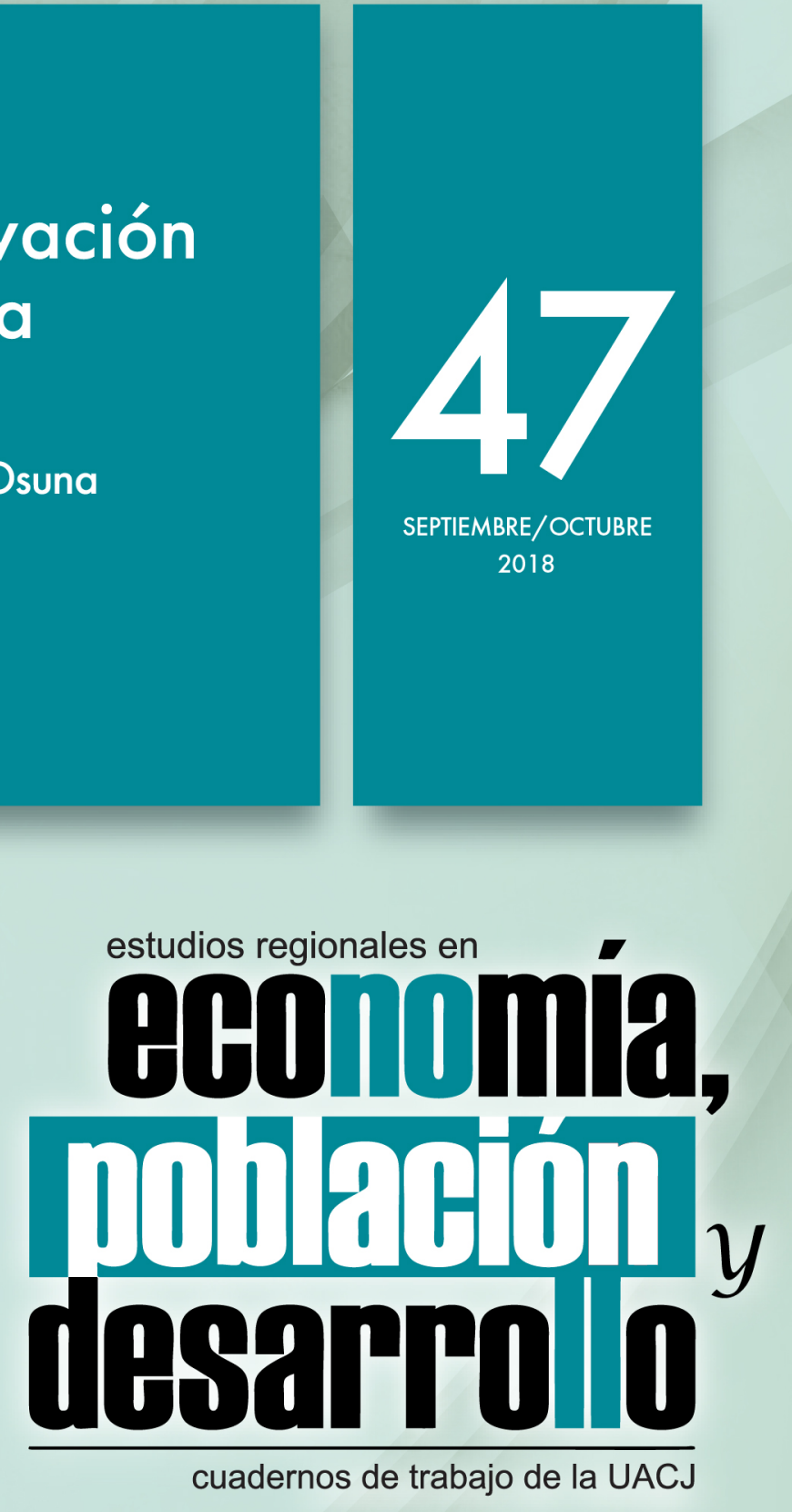


\title{
Ciencia, tecnología e innovación en México: un análisis de la política pública
}

\author{
Claudia Díaz Pérez y Moisés Alejandro Alarcón Osuna
}




\title{
UNIVERSIDAD AUTÓNOMA DE CIUDAD JUÁREZ \\ Instituto de Ciencias Sociales y Administración
}

\author{
Cuerpo Académico de Estudios Regionales en \\ Economía, Población y Desarrollo
}

Lic. Ricardo Duarte Jáquez

Rector

M.C. David Ramírez Perea

Secretario General

Mtro. Juan Ignacio Camargo Nassar

Director del Instituto de Ciencias

Sociales y Administración

Mtro. Ramón Chavira Chavira

Director General de Difusión

Cultura y Divulgación Científica

Dr. Luis Enrique Gutiérrez Casas

Coordinador General de

Investigación y Posgrado

\section{Dr. Jaime Alberto Arellano Quiroga \\ Coordinador del Cuerpo Académico de \\ Estudios Regionales en Economía, Población y Desarrollo}

Director y editor

Dr. Luis Enrique Gutiérrez Casas

Comité editorial

Sección internacional

Dra. Sofía Boza Martínez (Universidad de Chile, Chile)

Dra. Olga Biosca Artiñano (Glasgow Caledonian

University, Reino Unido)

Dra. Ángeles Sánchez Díez (Universidad Autónoma de

Madrid, España)

Dr. Thomas Fullerton Mankin (University of Texas at

E1 Paso, Estados Unidos)

Dr. Adrián Rodríguez Miranda (Universidad de la

República, Uruguay)

Sección local

(Universidad Autónoma de Ciudad Juárez)

Dra. Myrna Limas Hernández

Dra. Ikuho Kochi

Dr. Raúl Alberto Ponce Rodríguez

Dr. Isaac Leobardo Sánchez Juárez

Dr. Héctor Alonso Barajas Bustillos

Diseño de cubierta Abigail Bautista
Estudios Regionales en Economía, Población y Desarrollo. Cuadernos de Trabajo de la UACJ ISSN 2007-3739

Número 47. Septiembre/Octubre 2018

Ciencia, tecnología e innovación en México:

un análisis de la política pública

Claudia Díaz Pérez y Moisés Alejandro Alarcón Osuna

Universidad Autónoma de Ciudad Juárez

Estudios Regionales en Economía, Población y Desarrollo. Cuadernos de Trabajo de la UACJ

Año 8, No. 47 septiembre-octubre 2018, es una publicación bimestral editada por la Universidad Autónoma de Ciudad Juárez a través del Cuerpo Académico de Estudios Regionales en Economía, Población y Desarrollo del Instituto de Ciencias Sociales y Administración. Redacción: Avenida Universidad y H. Colegio Militar, Zona Chamizal s/n., C.P. 32300, Ciudad Juárez, Chihuahua, México. Teléfonos: (656) 688-38-00, ext. 3792. Correo electrónico: lgtz@uacj.mx.

Editor responsable: Luis Enrique Gutiérrez Casas. Reserva de derechos al uso exclusivo No. 04-2011-021713353900-102. ISSN 2007-3739, Impresa por Studio Los Dorados, calle Del Campanario, número 820-2, Santa Cecilia, C.P. 32350, Cd. Juárez, Chihuahua. Distribuidor: Subdirección de Gestión de Proyecto y Marketing Editorial. Ave. Plutarco Elías Calles 1210, Foviste Chamizal, C.P. 32310, Ciudad Juárez, Chihuahua. Este número se terminó de imprimir el 15 de julio, 2018 con un tiraje de 120 ejemplares.

Los ensayos publicados son responsabilidad exclusiva de sus autores. Se autoriza la reproducción total o parcial bajo condición de citar la fuente.

\section{Registrada en: EBSCO RePHC}

Publicación afiliada a la Red Iberoamericana de Estudios del Desarrollo

Universidad Autónoma de Ciudad Juárez

Ave Plutarco Elías Calles 1210

Foviste Chamizal, C.P. 32310

Ciudad Juárez, Chihuahua, México

www.uacj.mx

(C) Universidad Autónoma de Ciudad Juárez 
Estudios Regionales en Economía, Población y Desarrollo. Cuadernos de Trabajo de la UACJ, Universidad Autónoma de Ciudad Juárez , número 47, septiembre - octubre de 2018, ISSN 2007-3739, pp. 3-33, México.

\title{
Ciencia, tecnología e innovación en México: un análisis de la política pública
}

\author{
Claudia Díaz Pérez * y Moisés Alejandro Alarcón Osuna **
}

\begin{abstract}
Resumen
El propósito de este trabajo es explorar el efecto que han tenido las políticas de Ciencia, Tecnología e Innovación (CTeI) en la capacidad de innovación del país (CI) en el periodo 2007-2011. Este periodo se selecciona porque permite analizar las nuevas políticas de CTeI centradas en incentivar la demanda y las viejas politicas de CTeI, más orientadas a consolidar la oferta, instrumentadas por el gobierno federal. Este análisis se realiza con datos en panel que equiparan las 32 entidades federativas, en dicho periodo, con regiones ya que se parte de la perspectiva de los sistemas regionales de innovación (SRI). Las variables incorporadas al modelo son: miembros del SNI, becarios CONACYT, articulos por investigador; miembros del Registro Nacional de Ciencia y Tecnología (RENIECYT), Inversión Extranjera Directa en cada estado, empresas certificadas. Las variables que permiten diferenciar a los estados son: el Producto Interno Bruto (PIB) estatal, la población, y la matrícula de educación superior. Los resultados señalan que la variable becarios CONACYT no tiene efecto en la capacidad innovadora. Otro hallazgo interesante sugiere que al considerar las políticas centradas en la oferta, y las políticas que impulsan la demanda, son éstas últimas las que tienen mayor relevancia en relación a la CI, ya que el impacto del personal RENIECyT es mayor al generado por los investigadores del SNI.
\end{abstract}

Palabras clave: Tecnología, innovación, México.

\begin{abstract}
This study aims to explore the impacts that have had science, technology and innovation (ST\&I) policies on regional innovation capacity in the period 2007-2011. The new and old ST\&I policies implemented by the government are compared. The study uses a panel data analysis, which takes into account the 32 Federal States in that period as equivalent to regions. The analytical perspective is derived from Regional Innovation Systems (RIS). The main variables included on the model are: number of researchers recognized by the National Research System, CONACYT scholarship holders, articles per researcher; members of the National Register of Science and Technology (RENIECYT) Foreign Direct Investment in each state, certified companies. The variables that distinguish states are the state gross domestic product (GDP), population, and enrollment in higher education. The results indicate that the variable CONACYT scholarship holders have no effect on the innovative capacity. Another interesting finding suggests that when considering policies focused on supply, and policies that drive demand, it is the latter that is most relevant in relation to the IC, as the impact of staff RENIECyT is greater than that generated by researchers from National Research System.
\end{abstract}

Keywords: Technology, innovation, Mexico.

JEL Classification: 031, 033, 038.

- Recibido en: Abril de 2018.

- Aprobado en: Junio de 2018.

* Profesora-investigadora del Departamento de Producción Económica de la Universidad Autónoma Metropolitana, Unidad Xochimilco (México). Correo electrónico: cdiaz@correo.cua.uam.mx.

** Profesor-investigador de la Facultad de Ciencias Económicas y Sociales de la Universidad Autónoma de Sinaloa, Campus Culiacán (México). Correo electrónico: malarcon@uas.edu.mx. 


\section{$\rightarrow$ 1. Introducción.}

Esta investigación tiene como objetivo explorar en qué medida la política de ciencia, tecnología e innovación (CTeI) tiene efectos en la capacidad innovadora, medida a través del número de solicitudes de patentes en los diferentes estados del país. La capacidad innovadora se analiza particularmente en relación al potencial que tiene cada estado para que, dado el conjunto de insumos a la ciencia y la tecnología (CyT), los sistemas regionales de innovación (SRI) en México puedan evaluar su desempeño. La definición tradicional de innovación la considera como una invención que tiene éxito de mercado. En este marco, la variable dependiente muestra el esfuerzo desarrollado a nivel estatal para generar innovaciones a través del número de solicitudes de patentes ya que muchos estados no tienen patentes concedidas. Sin embargo, se considera que las patentes solicitadas muestran bien lo que se conceptualiza como esfuerzo tecnológico o capacidad innovadora. El esfuerzo tecnológico es el conjunto de procesos que se concretan en algún tipo de propiedad intelectual que, aun cuando no concluyan con el otorgamiento de patentes, muestran las actividades de $\mathrm{I}+\mathrm{D}$, las capacidades tecnológicas y los procesos administrativos de soporte que se despliegan (Soria, 2015).

Este trabajo se integra considerando dos de las perspectivas de análisis más relevantes de los estudios de la innovación. Primero, la propuesta derivada de los estudios de política científica e innovación (Martin, 2012), que permite definir el marco analítico para evaluar las políticas de CTeI. Segundo, los estudios enmarcados en la perspectiva de los sistemas de innovación. En esta línea de estudios, las características locales, los ambientes regionales, las organizaciones y políticas institucionales son factores determinantes de la capacidad de innovación (Ivanova y Leydesdorff, 2014; Lundvall, 1992; Nelson, 1993; Freeman, 1987). En particular, esta perspectiva permite identificar a las regiones como espacios significativos en donde las políticas de CTeI tienen un rol en el desarrollo de la innovación tecnológica y por lo tanto en la generación de capacidades de innovación. El periodo de análisis considerado es del 2007 al 2011 ya que permite contrastar medidas asociadas tanto a las políticas centradas en incentivar la oferta de tecnología como aquellas orientadas al desarrollo de la demanda, con el propósito de explorar cuáles tienen mayor peso en explicar la capacidad innovadora de cada región. La capacidad innovadora se analiza a partir de dos variables proxy: el número de patentes solicitadas por estado y las patentes solicitadas por millón de habitantes. Esta última variable permite incorporar las características particulares de cada región del país.

La perspectiva de los sistemas regionales de innovación permite delimitar el efecto de las políticas de CTeI para identificar si efectivamente existen impactos diferenciados por las características específicas de los estados en el país. Este abordaje regional facilita también el acceso a la información, ya que ese es uno de los aspectos más débiles en la evaluación de las políticas de CTeI 
en el país, a pesar del trabajo sistemático de organismos como el Foro de Ciencia y Tecnología y el propio CONACYT.

Los estudios de la innovación, y particularmente la perspectiva de los sistemas de innovación, explican la naturaleza sistémica de la innovación, así como los diversos procesos (económicos, sociales, políticos, etc.) que delinean el desarrollo, difusión y uso de la innovación (Edquist, 1997). Otro aspecto fundamental que abordan estos estudios, es la naturaleza evolutiva de la innovación asociada a las políticas, a las instituciones locales, y a la infraestructura científica, tecnológica e industrial. Asimismo, al observar que las actividades innovadoras no están distribuidas de manera uniforme, cobra mayor relevancia el análisis de la proximidad espacial y la concentración (Asheim y Gertler, 2005). La concentración del conocimiento, y por tanto el desarrollo de capacidades de absorción llevan a primer plano la importancia de las localidades y su relación con regiones similares, a nivel nacional y global (Basile, Capelio y Caragliu, 2012). En este contexto el análisis desde la perspectiva de los SRI permite articular el análisis de la estructura productiva local y la configuración institucional (Asheim y Gertler, 2005). Este nivel intermedio entre la empresa y el nivel nacional es en donde se identifica el nivel regional que articula y, a la vez, permite observar por qué hay regiones más competitivas que otras (Abdel Musik, 2000). Se ha señalado también que el concepto de región es ambiguo, pero que existen factores comunes que facilitan su análisis: i) la existencia de un marco institucional y cultural de soporte; ii) el aprendizaje como un proceso localizado; iii) la innovación como un proceso de aprendizaje interactivo, en donde la cercanía favorece la interacción; y iv) la aglomeración como base más eficiente para el aprendizaje interactivo (Dutrenit, 2009).

Por otro lado, y más explícitamente a partir del Programa Especial de Ciencia y Tecnología 2001-2006 México ha impulsado una política de CTeI y programas específicos que promueven la innovación tecnológica y a las empresas como actores esenciales para la competitividad y desarrollo económico. Se han creado programas de apoyo a la innovación orientados a las empresas (Corona et al., 2013), pero se han mantenido programas previos como las becas de posgrado CONACYT y el Sistema Nacional de Investigadores (SNI), programas creados en orientaciones de política previas más centradas en la oferta de conocimiento. El análisis de las diferencias entre estas políticas y programas de naturaleza diferente, pero complementaria, así como el efecto que tienen en las regiones o estados del país, es el eje de esta propuesta.

En este trabajo se propone analizar el impacto que han tenido las políticas de CTeI para impulsar la capacidad innovadora en las regiones del país para el periodo 2007-2011. En este periodo se pueden evaluar los efectos de las nuevas políticas de CTeI instrumentadas por el gobierno federal (o políticas centradas en la demanda), respecto a las políticas orientadas a la oferta de CyT. Se propone un análisis con datos en panel, que tomen en cuenta a las 32 entidades federativas en 
el periodo señalado. Se exploran mediante la instrumentación de efectos aleatorios los efectos de la política de CTeI en la capacidad innovadora considerando la dinámica local que se ha seguido, y agrupando las entidades federativas de acuerdo a su rendimiento en relación a las políticas de $\mathrm{CTeI}^{1}$. La hipótesis central es que las nuevas políticas de CTeI han tenido un impacto positivo en la capacidad de innovar tecnológicamente en las regiones del pais, sobre todo al incorporar personal de empresas a las actividades de desarrollo tecnológico e innovación. Las políticas de CTeI centradas en la oferta de CyT se evalúan a partir del número de miembros del SNI, del número de becarios CONACYT y del número de artículos por investigador. Las políticas de CTeI orientadas a la demanda de tecnología se analizan a partir de los miembros del Registro Nacional de Ciencia y Tecnología (RENIECYT) del CONACYT, de la Inversión Extranjera Directa (IED) en cada estado, y del número de empresas certificadas. Las variables que permiten diferenciar a los estados son: el Producto Interno Bruto (PIB) estatal, la población, y la matrícula de educación superior. Estas variables proxy permiten inferir las capacidades desarrolladas en los estados a partir de los recursos humanos, el valor de su economía y la estructura productiva local, ya que el número de empresas con certificación ISO se relaciona con la consolidación de las cadenas productivas y la vinculación de las empresas locales con multinacionales (Abdel Musik, 2000).

El artículo se organiza en seis apartados: En la introducción, además del objetivo, se presenta el problema de investigación y las principales limitaciones del estudio. En la segunda sección se analiza la evolución de las políticas de CTeI en México y el desempeño y evolución de los principales indicadores, particularmente de aquellos centrados en la innovación. Se describe el papel del CONACYT como distribuidor de recursos económicos, la innovación en los sectores productivos, y la relación entre patentes e innovación. En la tercera sección se plantea el marco analítico donde se abordan: (i) Los estudios de políticas de CTeI que dan la pauta para esta investigación, y las principales dificultades que presentan. (ii) Los sistemas de innovación como marco para el análisis de las dinámicas regionales y su desempeño; (iii) El análisis de la capacidad innovadora como la base para impulsar el desarrollo de la innovación tecnológica, y (iv) Las patentes como indicador de la capacidad innovadora desde la perspectiva del cambio tecnológico y de los sistemas nacionales de innovación (SNInn). Se analizan también las limitaciones que tienen como indicador estas variables. En la cuarta sección se presentan los datos y el modelo de contrastación. En la quinta sección se analizan los resultados y las variables más significativas del modelo, y se interpretan a partir de la literatura del campo. Finalmente se presentan las conclusiones.

1 En estudios recientes, realizados por el Foro Consultivo Cientifico y Tecnologico en 2014, se destaca que el Gasto Federal en Ciencia y Tecnología, tarda aproximadamente 4 años en generar sus mayores impactos sobre la productividad de las personas (Santiago y Natera, 2014). 


\section{$\rightarrow$ 2. Las políticas de CTeI en México.}

Este apartado sintetiza las principales propuestas identificadas tanto en la política de CyT centrada en la oferta, como la orientada a promover la demanda tecnológica. Con este propósito, además de describir las principales propuestas de la política, se presenta el comportamiento de los principales factores que dan cuenta del desempeño actual en relación a la innnovación tecnológica en el sector empresarial, los recursos destinados por el CONACYT, los recursos humanos especializados, así como la información de patentamiento. En conjunto, esta información permite explorar el panorama mexicano en el ámbito de la CTeI.

\subsection{La evolución de las políticas de ciencia y tecnología en México.}

En México, el primer plan de largo aliento para planificar la ciencia y tecnología se formalizó en 1976. Desde entonces se buscaba que el gobierno llegara, en un plazo de 25 años, a invertir el 1\% de su PIB (Díaz, 2002). Esta meta se plantea también en el PECITi 2001-2006, en el sexenio del presidente Fox. José Sarukhán señalaba, a finales de los noventa, que el CONACYT había impulsado el desarrollo de la política de ciencia y tecnología, particularmente a través de proyectos de investigación, desarrollo de infraestructura, entrenamiento de personal y el establecimiento de centros de investigación fuera de la capital del país (Sarukhan, 1989).

La política de CyT en México, más recientemente llamada política de CTeI, inició su transición hacia la perspectiva de la demanda desde la década de los ochentas. El Programa Nacional de Desarrollo Tecnológico y Científico (1984-1988) fue el primero en establecer medidas inductivas para la participación del sector privado. Se orientó principalmente hacia lo que se llamó autodeterminación tecnológica, buscando la participación de las empresas y el financiamiento complementario. Este cambio se consolidaría en los programas de los sexenios posteriores (90-94 y 95-00), donde el énfasis estaría en la vinculación, la racionalización de recursos y la participación del sector privado (Díaz, 2002; 2007). En esta década (noventas), los programas de apoyo se orientaron de manera importante hacia las regiones y hacia el impacto en necesidades específicas en las mismas (Casas, 2001). Esta tendencia se fortaleció en la década siguiente a través de los respectivos programas de ciencia y tecnología que ya hacen referencia a la innovación y otorgan un papel explícitamente preponderante al sector privado. En este periodo los programas de financiamiento del CONACYT incentivan la participación de las empresas, la creación de negocios, la creación de invenciones y su registro como patentes, la innovación tecnológica en productos y procesos a través de diferentes instrumentos, entre otros (Díaz, 2013). Sin embargo, a lo largo del tiempo prevalecen indicadores de ambos tipos de políticas. Esto es, coexisten programas que se crearon en un contexto de políticas de oferta de ciencia y tecnología con programas orientados a fortalecer, principalmente, 
la demanda de tecnología y la consolidación de las empresas tecnológicas.

Leyva y Barraza (2007) hace un análisis de la política de ciencia y tecnología en México en el periodo del 2001 al 2006 e identifica que el principal problema que tiene es la falta de elementos para su instrumentación, por lo que se queda en el nivel de la planeación. Señala también que la Ley de Ciencia y Tecnología obliga a los estados a incrementar su gasto en ciencia y tecnología hasta el $1 \%$ para el 2006, meta que no se cumplió. Los principales indicadores que la política plantea son el financiamiento (inversión nacional en CyT como porcentaje del PIB, gasto en I+D como porcentaje del PIB, participación del sector productivo en el gasto en I+D); la formación de recursos humanos (número de personas dedicadas a $\mathrm{I}+\mathrm{D}$, número de personal dedicado a $\mathrm{I}+\mathrm{D}$ por cada 1000 habitantes de la PEA, porcentaje de investigadores en el sector productivo, formación de doctores por año e incluye algunos); indicadores de competitividad (posición mundial en infraestructura científica, posición mundial en infraestructura tecnológica).

La política de $\mathrm{CTeI}$ se ha ido consolidando, al menos en términos de su planeación, a lo largo de tres décadas pero es hasta el 2002 cuando se orienta explícitamente a la promoción de la innovación, principalmente tecnológica. Sin embargo, es claro que prevalecen programas que responden a la perspectiva de la oferta de conocimiento, por lo que la pregunta pertinente que se deriva es ¿Cuáles políticas tienen un mayor impacto en la promoción de la capacidad innovadora y por lo tanto de la innovación tecnológica?

\subsection{Las políticas de CTeI y la innovación tecnológica en las regiones de México.}

A excepción del Distrito Federal, el Estado de México, Jalisco, Nuevo León y Puebla, las regiones mexicanas tienen un bajo desempeño tecnológico y un relativamente escaso desarrollo económico. Este fenómeno puede explicarse en buena medida al hecho de que las políticas de CTeI se han diseñado como un apéndice de las políticas industriales (Mora y Soria, 2012; Corona et al., 2013). Este hecho ha generado que las políticas de CTeI jueguen un papel secundario en el crecimiento económico, relegándolas a un plano pasivo, donde el avance científico está centrado en la oferta de servicios y bienes tecnológicos, por ello, impulsar un cambio tecnológico mediante los instrumentos propuestos dentro de perspectivas como los SRI en México, ha sido resultado de esfuerzos aislados que dependen en buena medida de los gobiernos locales y/o asociaciones empresariales (Díaz et al., 2011).

Si se analiza el nivel de innovación del sector empresarial, el estrato de la gran empresa juega un papel importante en la cantidad de innovaciones, (ver cuadro 1). El resto de estratos de empresa tienen una escasa capacidad para competir a partir de la innovación, en dicho estudio se intenta aproximar a una medición de las capacidades de Ciencia, Tecnología e Innovación en México (Dutrenit y Ramos, 2012). 
Cuadro 1

Distribución de empresas innovadoras por tipo de producto 2006

\begin{tabular}{|c|c|c|c|c|}
\hline & & \multicolumn{3}{|c|}{ Ventas del total de empresas (\%) } \\
\hline $\begin{array}{c}\text { Tamaño de empresa (número } \\
\text { de empleados) }\end{array}$ & $\begin{array}{c}\text { \% de empresas } \\
\text { innovadoras }\end{array}$ & $\begin{array}{c}\text { Productos } \\
\text { tecnológicamente } \\
\text { nuevos }\end{array}$ & $\begin{array}{c}\text { Productos } \\
\text { tecnológicamente } \\
\text { mejorados }\end{array}$ & $\begin{array}{c}\text { Productos no } \\
\text { modificados }\end{array}$ \\
\hline $50-100$ & 21.8 & 17.5 & 30.4 & 52.1 \\
\hline $101-250$ & 28.8 & 36.5 & 27.2 & 36.3 \\
\hline $251-500$ & 27.7 & 20.5 & 26.1 & 53.4 \\
\hline $501-750$ & 26 & 25.9 & 15 & 59.1 \\
\hline 751 o más & 20.1 & 34.7 & 42.7 & 22.6 \\
\hline \% del total de empresas & 24.9 & 31.8 & 36.4 & 31.8 \\
\hline
\end{tabular}

Fuente: Dutrenit y Ramos (2012), con datos tomados de la encuesta nacional de Investigación y Desarrollo de CONACYT.

La proporción de empresas innovadoras en México representan casi la cuarta parte del total de empresas, de las cuales al menos dos tercios son empresas que generan productos nuevos o mejorados. Es difícil saber qué tan innovadoras son las empresas de tamaño micro, pequeñas y medianas (MiPYMES). Algunos estudios han estimado que del total de empresas innovadoras (10\% a nivel México) ${ }^{2}$, las de tamaño MiPYME son alrededor del 97\% para los años 2004 y 2009 (Alarcón y Díaz, 2014).

En cuanto a los recursos destinados por parte del CONACYT para ciencia y tecnología en 2009 (Dutrenit y Ramos, 2012), se encuentra la siguiente distribución: 5\% para ciencia básica, 6\% de gastos operativos, $8 \%$ para ciencia aplicada, 19\% para innovación, y $62 \%$ para formación de recursos humanos. Esta distribución muestra que la principal estrategia en políticas de CTeI es generar una masa crítica de recursos humanos capaces de generar el entorno innovador para el sector productivo, ya que la mayor parte se invierte en recursos humanos como científicos, tecnólogos y estudiantes de posgrado, lo cual puede ser visto como una política lineal y centrada en la oferta. En el 19\% para innovación, se contempla la participación de empresas privadas, principalmente, mediante los programas concursables y distribuidos a través del RENIECYT ${ }^{3}$, constituyendo de esta forma un enfoque evolutivo en la generación de políticas de CTeI (Dutrenit y Ramos, 2012). El primer enfoque descrito impulsa la innovación de manera primordial a través de la inversión en ciencia básica y avanzada, sin que necesariamente se involucrara al sector privado con sus nece-

2 Se toma a las Empresas de Base Tecnológica, como las empresas innovadoras, y el cálculo es realizado a partir de los censos económicos 2004 y 2009.

3 RENIECYT, tiene como objetivo mantener una base de datos de las instituciones, centros, organizaciones, empresas o personas físicas que realicen actividades científicas y tecnológicas, constituyéndose como un prerrequisito para las personas físicas o morales interesadas en recibir los beneficios o estímulos de cualquier tipo, derivados de los ordenamientos federales aplicables a estas actividades. 
sidades específicas de innovación a nivel regional. Esta formación de una masa crítica en CTeI, se describe en el cuadro 2, el cual muestra a los investigadores (SNI) por millón de habitantes, personal RENIECYT por millón de habitantes, becas CONACYT otorgadas por millón de habitantes, y las matrículas de licenciaturas y posgrados de universidades y tecnológicos en el país por millón de habitantes.

Cuadro 2

\section{Miles de personas por personal involucrado en actividades de CTeI} 2007-2012

\begin{tabular}{|c|c|c|c|c|c|}
\hline Año & SNI & RENIECYT & Matrícula Licenciatura & Matrícula Posgrado & Beca conacyt \\
\hline 2007 & 94.13 & 31.65 & $20,566.37$ & $1,488.16$ & 148.44 \\
\hline 2008 & 118.45 & 39.00 & $21,068.55$ & $1,578.59$ & 159.41 \\
\hline 2009 & 120.79 & 45.78 & $21,421.70$ & $1,658.37$ & 184.65 \\
\hline 2010 & 131.45 & 59.62 & $22,333.16$ & $1,735.50$ & 237.54 \\
\hline 2011 & 139.43 & 66.36 & $23,102.82$ & $1,816.09$ & 257.08 \\
\hline 2012 & 145.70 & 59.99 & $24,166.20$ & $1,975.62$ & 301.83 \\
\hline
\end{tabular}

Fuente: Elaboración propia con datos de ANUIES, CONAPO y Foro Consultivo Cientifico y Tecnológico.

Se observa una tendencia creciente en el número de científicos, personal dedicado a desarrollo tecnológico, matrículas de licenciatura y posgrado, así como del otorgamiento de becas para estudiar posgrados por miles de habitantes en México, dado que ha disminuido el número de personas por personal involucrado en actividades de CTeI. Sin embargo, aún no se puede llegar a una conclusión respecto de la formación de una masa crítica, la distribución de gastos de CONACYT y la distribución de empresas innovadoras, ya que hace falta evaluar el impacto de la CTeI en el desempeño económico nacional, a través del PIB. Es por ello que se muestran en el cuadro 3 algunos indicadores de la capacidad innovadora en México.

En el cuadro 3 se observa que es difícil describir una relación entre las patentes y el PIB, aunque existe una tendencia creciente en ambos rubros con un ligero descenso entre los años 20092010 en el número de patentes otorgadas y el PIB, es difícil establecer si existe una relación entre ambos. No obstante la estadística es útil, ya que permite suponer una relación tenue entre ambas variables. 
Cuadro 3

\section{Capacidad innovadora en México y su relación con el funcionamiento económico}

\begin{tabular}{|c|c|c|c|c|}
\hline Año & Patentes solicitadas & Patentes otorgadas & Solicitud patente $\mathrm{P} / \mathrm{H}^{*}$ & PIB \\
\hline 2007 & 640 & 198 & 4.50 & $11,778,875$ \\
\hline 2008 & 675 & 197 & 4.89 & $11,941,200$ \\
\hline 2009 & 815 & 249 & 5.60 & $11,374,629$ \\
\hline 2010 & 939 & 248 & 6.55 & $11,965,979$ \\
\hline 2011 & 1058 & 287 & 7.45 & $12,435,054$ \\
\hline 2012 & 1284 & 281 & 8.54 & $12,935,717$ \\
\hline
\end{tabular}

Fuente: Elaboración propia con datos del IMPI e INEGI. *Se toman las solicitudes de patente por millón de habitantes. PIB a precios de 2003.

Las diversas regiones del país tienen sus propias dinámicas locales en donde se concretan las interrelaciones específicas entre las instituciones y empresas que se establecen en la zona. Dadas las diferencias esperadas en las regiones o estados del país, es importante mostrar el desempeño que tienen las entidades federativas en el patentamiento. Aunque el periodo de análisis en este estudio es 2005-2011, el cuadro 4 muestra las patentes solicitadas en el periodo 2005-2012, en cada una de las entidades federativas. Se observa una clara concentración en el Distrito Federal y en los estados que presentan el mayor dinamismo económico del país.

Como se observa en el cuadro 4 el DF, Nuevo León, Jalisco y Estado de México mantienen los primeros lugares tanto en patentes solicitadas como en patentes otorgadas. Un segundo grupo de estados tiene más de 10 patentes otorgadas, y después de ellos existen 15 entidades que no logran obtener más de 8 patentes en un periodo de 8 años, aunque su actividad inventiva muestre que se solicitan más de 20 patentes en ese periodo. Este desempeño diferenciado en relación a la referencia geográfica requiere una exploración de los factores asociados al mismo, como un elemento para el análisis de las políticas de CTeI y su relación con la capacidad innovadora de las diversas regiones del país. 


\section{Cuadro 4}

\section{Patentes solicitadas y otorgadas por entidad federativa}

2005-2012

\begin{tabular}{|c|c|c|}
\hline Entidad & Patentes otorgadas & Patentes solicitadas \\
\hline Zacatecas & 0 & 16 \\
\hline Campeche & 1 & 29 \\
\hline Nayarit & 1 & 6 \\
\hline Guerrero & 2 & 13 \\
\hline Aguascalientes & 2 & 39 \\
\hline Chiapas & 2 & 39 \\
\hline Tlaxcala & 2 & 27 \\
\hline Hidalgo & 3 & 51 \\
\hline Quintana Roo & 3 & 19 \\
\hline Baja California Sur & 3 & 17 \\
\hline Durango & 6 & 30 \\
\hline Oaxaca & 7 & 25 \\
\hline Sinaloa & 7 & 94 \\
\hline Colima & 7 & 26 \\
\hline Baja California & 8 & 81 \\
\hline San Luis Potosí & 10 & 51 \\
\hline Yucatán & 11 & 94 \\
\hline Tabasco & 13 & 41 \\
\hline Michoacán & 14 & 56 \\
\hline Tamaulipas & 17 & 97 \\
\hline Sonora & 18 & 140 \\
\hline Veracruz & 21 & 126 \\
\hline Chihuahua & 33 & 170 \\
\hline Coahuila & 37 & 212 \\
\hline Guanajuato & 47 & 231 \\
\hline Morelos & 49 & 179 \\
\hline Puebla & 66 & 318 \\
\hline Querétaro & 68 & 223 \\
\hline Edo. Mexico & 126 & 558 \\
\hline Jalisco & 128 & 578 \\
\hline Nuevo León & 278 & 853 \\
\hline $\mathrm{DF}$ & 730 & 2120 \\
\hline
\end{tabular}

Fuente: Elaboración propia con datos de IMPI. 


\section{$\rightarrow$ 3. El análisis de la innovación tecnológica.}

En este apartado se exploran algunas de las propuestas más significativas para abordar el análisis de la innovación tecnológica, así como su conceptualización. Con este propósito se revisan algunas propuestas y las principales dificultades en el análisis de las políticas de CTeI. También, las aportaciones derivadas de la perspectiva de los sistemas de innovación, particularmente de los SRIs. Asimismo se exploran las nociones de capacidad innovadora que permiten configurar el concepto de innovación tecnológica usado en esta investigación. Finalmente se analiza el uso de las patentes como indicador de la capacidad innovadora desde la perspectiva del cambio tecnológico como desde los sistemas regionales de innovación.

\subsection{El análisis de la política de CTeI.}

La perspectiva de los sistemas de innovación ha sido una de las más fructíferas en la maduración del campo del análisis de las políticas de ciencia y los estudios de la innovación. La perspectiva evolutiva, y el abordaje institucional ha permitido una profundización en la comprensión de la relación entre políticas e innovación (Martin, 2012). El diseño institucional y la participación de los gobiernos en la promoción de la producción, difusión y el uso del conocimiento científico y técnico han sido fundamentales para el logro de los objetivos nacionales. Sin embargo, uno de los retos principales ha sido la medición del impacto de estas políticas (Lundvall y Borrás, 2006). Las políticas se establecen tanto a nivel nacional como regional, sectorial, etc. o en una combinación de niveles. Sin embargo, el análisis de sus efectos presenta diversas dificultades.

Cotgreave y Davies (2005) señalan la importancia de las políticas nacionales de ciencia, tecnología e ingeniería para el desarrollo económico de Europa. Identifican un conjunto de indicadores para evaluar el éxito de la política nacional: la inversión pública en $\mathrm{I}+\mathrm{D}$, la estructura de las carreras científicas, la enseñanza de las ciencias, la inversión privada en $\mathrm{I}+\mathrm{D}$ y el uso de la ciencia para el desarrollo de políticas públicas. Por su parte, Brancati et al (2000) han señalado que a nivel regional, las políticas e instrumentos para la promoción de I+D y la innovación son fundamentales para que el gobierno oriente el desarrollo tecnológico regional. El financiamiento para infraestructura, entrenamiento de alto nivel, difusión tecnológica y el desarrollo tecnológico de las PYMES resultan muy importante siempre y cuando estén acompañados de estrategias locales. Por el contrario, la falta de coordinación, la debilidad de las herramientas de política a nivel regional y la ausencia de beneficiarios involucrados directamente tiene un impacto negativo en el desarrollo de capacidades tecnológicas. Este estudio identifica que la política pública para impulsar la I+D está diseñada desde la perspectiva de la oferta, principalmente a través de bolsas de recursos económi$\cos$ (Brancati et al, 2000). 
A partir de los ochenta, los gobiernos se concentran en consolidar la ciencia y la tecnología como estrategia de desarrollo económico. Los programas que se implementan están orientados a atraer, nutrir y desarrollar industrias de alta tecnología (Melkers, 2004). Feller (2007) señala que la educación superior fue el primer enfoque de la política de desarrollo de la ciencia y la tecnología. El cambio hacia el impulso de la innovación industrial ocurre a finales de los setenta, a iniciativa de la OCDE. Para Molas-Gallart y Davies (2006) las políticas de ciencia y tecnología orientadas a impulsar la oferta incluyen nuevo conocimiento científico y/o técnico que es resultado de actividades de investigación originales realizadas comúnmente en laboratorios de I+D y/o universidades. El lado de la demanda se caracteriza por que las nuevas tecnologías, productos, procesos y servicios se derivan de las demandas del Mercado (actuales o percibidas).

Entre las principales críticas al uso de indicadores cuantitativos de nivel macro, como el gasto gubernamental en $\mathrm{I}+\mathrm{D}$ es que dicen poco sobre los vínculos y los impactos observados en el desempeño, y solo pueden evaluarse en el largo plazo (Molas-Gallart y Davies, 2006). Otra de las críticas señala que los indicadores cuantitativos dicen poco acerca de la calidad de la investigación por ejemplo, y que se usan de manera intercambiable cuando no lo son. Por ejemplo, el ingreso de investigación es un insumo más que una medida de desempeño (Donovan, 2007). También se ha planteado que las medidas típicas de desempeño de la CyT como patentes, publicaciones, citas son indicadores rezagados y no dan cuenta precisa de los avances en investigación (Mote et al, 2007). Por otro lado, también se ha resaltado que el capital humano, entrenamiento y la educación son una capacidad primaria para la construcción de capacidades tecnológicas e innovación. Por lo tanto, se puede suponer que los indicadores que muestran la evolución y consolidación de la educación son fundamentales para entender el desempeño del sistema de innovación (Hage et al, 2007). La evaluación de las políticas de CyT y particularmente de su impacto no es una tarea sencilla, particularmente cuando no se cuenta con bases de información homogéneas a lo largo del país, y cuando las capacidades y diferencias regionales son cruciales. Bajo estas limitaciones, en este ensayo, se seleccionan aquellos indicadores en los cuales había información suficiente para una comparación a nivel estatal, aunque puedan tener limitaciones como variables proxy de los fenómenos estudiados. Algunas de las medidas más usadas para su evaluación son: la inversión pública en $\mathrm{I}+\mathrm{D}$, la inversión privada, las patentes, el gasto en educación. Recientemente, y con la consolidación de la perspectiva de los sistemas de innovación, se empieza a evaluar el desarrollo de la innovación a partir de variables integradas como: la movilidad de la fuerza de trabajo nacional, la cooperación formal e informal en I+D, la difusión de la innovación, entre otras (Balzat y Hanusch, 2004).

\subsection{El análisis del desempeño de los sistemas de innovación.}

La perspectiva de los SRI enfatiza que los sistemas son no lineales y se caracterizan por 
la interrelación entre diversos actores y organizaciones (institutos de investigación, universidades, empresas, gobierno, etc.) en el nivel regional (Equist, 2006; Edquist y Hommen, 1999). En otro estudio, realizado para el caso mexicano, se señala la forma en que los SRI impulsan las derramas tecnológicas de las empresas trasnacionales, así como los procesos de aprendizaje tecnológico, acumulación de capacidades y creación de capacidades de absorción. Se destaca la importancia de los encadenamientos productivos de la trasnacional y los proveedores locales, así como la movilidad de capital humano, taxonomías que reflejen niveles mínimos de conocimiento y niveles de capacidades innovadoras. El estudio, describe que los SRI están conformados como redes entre diversas instituciones que generan innovaciones, impulsando el mejoramiento productivo y la competitividad de las empresas, y reflejando el cambio tecnológico de una región (Dutrénit, 2009).

Asimismo, Dutrenit y Zuñiga (2013) establecen que las políticas de CTeI son un instrumento para impulsar el desarrollo de los SRI, y consolidarlos en el largo plazo. En esta línea se identifica el trabajo seminal de Asheim (1998) en cuya clasificación de SRI, señala que los sistemas de innovación regional en red se diseñan a través de la política pública que impulsa el fortalecimiento particular de una región.

Esta perspectiva enfatiza el diseño institucional como una herramienta clave en donde las políticas públicas permiten definir y reorientar las estrategia a nivel regional para el crecimiento económico (Geels, 2014). En ese marco, el sector público o el gobierno, como instancia coordinadora en el diseño de políticas públicas ${ }^{4}$, impulsa el establecimiento de políticas de apoyo para el desarrollo de los SRI (Corona et al, 2013). En particular, la política de CTeI se constituye como un instrumento para revitalizar la dinámica y competitividad en localidades, regiones y a nivel nacional. Es por ello que las políticas de CTeI no se pueden limitar solo a la definición de estrategias, prioridades e instrumentos para estimular la innovación. Además deben estar acompañadas de los recursos necesarios para la formación, fomento, transmisión, absorción y explotación del conocimiento social y económicamente útil, que sea capaz de generar una masa crítica en CTeI (Corona et al., 2011), así como de los elementos para evaluar su funcionamiento. Como se deriva de esta sección la política de $\mathrm{CTeI}$ es un instrumento central para fortalecer el desarrollo de SRI. En los países en donde la innovación no se ha consolidado, la capacidad innovadora permite estudiar el potencial que se tiene y por tanto establecer mecanismos de apoyo para su desarrollo en las regiones.

\subsection{La capacidad innovadora de las regiones.}

Un concepto que ha permitido estudiar si una región, localidad, sector, o empresa cuenta con los elementos para iniciar procesos de innovación es el de capacidad innovadora. Aunque esta

4 En México el Consejo Nacional de Ciencia y Tecnología (CONACYT), juega un papel central en los SRI, coordinando e implementando las políticas de CTI, y siendo el intermediario entre el gobierno y los científicos y el gobierno y las empresas (Dutrenit y Ramos, 2012). 
se ha estudiado principalmente para las empresas, se identifican algunos estudios que lo abordan a nivel regional.

Zawislak et al (2012) definen la capacidad de innovación como aquella que incluye la capacidad de absorción de nuevo conocimiento, así como la capacidad de adaptar y transformar una determinada tecnología e incorporarla a rutinas específicas que eventualmente generarán innovaciones y beneficios económicos. En esta misma línea, Ospina (2010) plantea que la capacidad de innovación se despliega a través de los procesos de creación, absorción, reconfiguración e integración del conocimiento. Para Neuman et al (2015), la capacidad innovadora se refiere al conjunto de actividades científicas, tecnológicas, organizativas, financieras, comerciales que llevan a una innovación. Se tipifica de esfuerzo cuando estas actividades no han conducido a la innovación en si misma. Sin embargo, se menciona también que, en general, la idea de capacidad innovadora o innovadora se aborda desde la perspectiva de las innovaciones de producto en lugar de una capacidad de innovación en general (Ibrahim et al, 2009).

También a nivel de la empresa, la propuesta de Boscherini y Yoguel (1996) es relevante, en tanto definen la capacidad innovadora como el potencial de idear, planear y realizar innovaciones a partir del uso de los conocimientos tecnológicos y organizativos formales e informales en la firma. Identifican además un conjunto de factores que permiten inferir la capacidad innovadora en las empresas: (i) la certificación en calidad, (ii) recursos humanos altamente calificados orientados a generar I+D, (iii) el rol de los nuevos productos en la facturación, (iv) el nivel de las actividades de innovación en sus diversas modalidades y, (v) la cooperación tecnológica con otras organizaciones. Esta propuesta permite aterrizar a nivel de variables e indicadores el concepto de capacidad innovadora y medirla a nivel empresas y organizaciones y transferirla al ámbito regional.

En el nivel regional destaca el planteamiento de Crescenzi (2005) que identifica como eje de la capacidad innovadora de las regiones a la inversión creciente y sistemática en capital humano y a las dinámicas locales. A nivel nacional, se identifica el estudio de Furman et al (2002) que conceptualiza la capacidad innovadora nacional como la habilidad de un país para producir y comercializar tecnología innovadora en el largo plazo, a partir de la solidez de la infraestructura tecnológica, el ambiente de innovación en los clusters y los vínculos que se producen entre ambos.

En este trabajo la capacidad innovadora de una región se considera como el conjunto de condiciones (educativas, económicas, de desarrollo de $\mathrm{I}+\mathrm{D}$, productivas, de recursos humanos) que permiten observar el potencial que tiene para crear innovación tecnológica. En este sentido, el concepto está estrechamente ligado a la propuesta de Soria (2015) de esfuerzo tecnológico que incluye las actividades que se requieren desplegar para concretar una solicitud de patente. Este debate se retoma en la siguiente sección. 


\subsection{Patentes, innovación tecnológica y capacidad innovadora.}

Las estadísticas de patentes son una fuente fundamental para mapear la evolución de los sistemas de innovación más que como indicadores de desempeño para evaluar la eficiencia de la política tecnológica (Lundvall y Borrás, 2006). Sin embargo, su uso como indicadores de innovación, de trayectorias tecnológicas, de actividad inventiva es muy amplio. Godin (2012) identifica dos grandes corrientes en los estudios de la innovación que han hecho un uso extenso de este indicador. Por un lado identifica a la tradición americana que analiza a la innovación como invención tecnológica introducida en los procesos de producción industrial. Esta corriente tiene su núcleo en la economía neoclásica, particularmente en los factores de producción. Por otro lado, está la tradición que concibe a la innovación tecnológica como la comercialización de invenciones, donde Freeman tiene a uno de sus primeros exponentes. Esta perspectiva tiene una preocupación explícita por el diseño de política de CyT para impulsar el desempeño económico.

Las patentes se consideran como un buen indicador tanto del cambio tecnológico como de la innovación tecnológica (Freeman, 1987; Lucas, 1988; Romer, 1989; Nelson, Winter and Schuette, 1976), ya que contienen una invención que ha pasado tanto el escrutinio de la oficina de patentes, como la inversión (tiempo, capacidades, económica) que ha realizado la persona o institución para el desarrollo de una nueva idea. De acuerdo con la OMPI los requisitos de patentabilidad son una solución nueva para algún producto, creatividad no obvia para una persona versada en la materia, que diferencia a la patente del modelo de utilidad, y finalmente una aplicación industrial o comercial.

Este nuevo conocimiento certificado por la oficina de patentes indica por un lado la presencia de una posible utilidad en los mercados (Griliches, 1990), y por otra parte, una medida de la capacidad tecnológica de las regiones (Mora y Soria, 2012), ya que mide la producción y acumulación de conocimientos, que a su vez representan los esfuerzos tecnológicos de las regiones. También es cierto que no todas las invenciones son patentables, ni todas las invenciones son patentadas, es por ello que se debe tomar con precaución a las patentes como un indicador del cambio tecnológico. Algunos de los estudios pioneros en la introducción de patentes, para explicar el rendimiento de la economía, han sido los estudios realizados por Schmookler en su disertación doctoral (1951), y posteriormente en sus aportes a revistas académicas (1952).

Pavitt (1998) enfatiza que las patentes son indicadores de la protección de innovaciones más que indicadores de procesos innovadores. Por ello se entiende que las patentes son el indicador que ha sido más estudiado para explicar el cambio tecnológico. Es por lo anterior que se han pro-

5 Esta relación se valida principalmente por la correlación lineal positiva que existe entre I+D y las patentes concedidas (Griliches, 1990), donde el experimento en una muestra de corte transversal demuestra una relación lineal positiva entre el logaritmo de las patentes concedidas y el logaritmo de los gastos en $\mathrm{I}+\mathrm{D}$ de las empresas, pero con un rendimiento decreciente de los gastos de I+D sobre las patentes concedidas (Griliches, 1994). 
puesto variables que reflejen las actividades de innovación, como los gastos en $\mathrm{I}+\mathrm{D}$, ya que además de las patentes, estos indicadores originados en los trabajos de Pavitt, Robson y Townsend (1987) y Edwards y Gordon (1984) documentan los fines que se le dan a los procesos de patentamiento.

No obstante a lo anterior, generalmente los autores coinciden en reconocer que el uso de las patentes como fuente de información asegura ventajas que superan sus limitaciones, que además brindan información tecnológica y con una estructura común. Que además, las patentes son la principal herramienta con la que se cuenta para descubrir la novedad que puede producir un cambio o un surgimiento abrupto de una tecnología durante una investigación (Diessler, 2010).

El concepto tradicional de innovación se ha definido como una invención con éxito de mercado. Sin embargo, se encuentran otras definiciones donde la innovación se refiere a alguna fase del ciclo que va de la investigación a la introducción al mercado de un nuevo producto o servicio (Molas-Gallart y Davies, 2006: 65). Tales consideraciones permiten establecer el supuesto de que las solicitudes de patente son una variable proxy de la capacidad innovadora, considerada como el esfuerzo que se despliega a nivel regional que incluye el financiamiento y las actividades de $\mathrm{I}+\mathrm{D}$, recursos humanos especializados, desarrollo del sector productivo, entre los más importantes. Dadas las limitantes en torno a las variables proxy sobre capacidad innovadora, en este trabajo se tomará como variable proxy la solicitud de patentes. La principal razón es que son los indicadores disponibles con una medición sistemática por parte de la oficina de patentes y que sintetizan tanto las actividades y la inversión en CyT a nivel estatal, como las realizadas por empresas, universidades e instituciones de investigación. En el siguiente apartado se detalla el método de contrastación y los datos utilizados para el modelo propuesto.

\section{$\rightarrow$ 4. Los datos y el método de contrastación.}

Dado el objetivo de estimar los efectos que han tenido las políticas de CTeI en el contexto de un SRI, se crea un panel de datos para las 32 entidades federativas en el periodo 2007-2012, en parte por la disponibilidad de datos y en parte por razones expuestas en los primeros apartados ${ }^{6}$. Se propone un análisis de regresión que tome en cuenta las prácticas del viejo modelo de CTeI como lo son las becas CONACYT y el sistema nacional de investigadores de $\mathrm{CONACYT}^{7}$, donde se contempla como variable proxy al número de investigadores por millón de habitantes, con la distin-

6 Es a partir de 2002, cuando se inicia una nueva política de CTeI por parte de CONACYT, respetando las viejas políticas de establecer un sistema nacional de investigadores y becarios de posgrado, y añadiendo la participación de empresas privadas en actividades de innovación, mediante su inclusión en proyectos de investigación con recursos financieros de CONACYT, y como toda política que toma tiempo en surtir efectos, se considera que los efectos más notorios deberían presentarse en periodos posteriores a 2005, es decir tres años después de implementada la nueva política. 
ción del sistema nacional de investigadores (el cual puede ser Candidato, Nivel I, Nivel II y Nivel III), a quienes se les dota de un estímulo por sus esfuerzos en actividades de investigación ya sea básica o aplicada la cual es revisada y avalada por sus pares. Por otro lado, se considera el número de becarios de posgrados CONACYT por millón de habitantes. Estos datos fueron tomados de los Diagnósticos Estatales de Ciencia, Tecnología e Innovación 2014, elaborados por el Foro Consultivo Científico y Tecnológico ${ }^{8}$.

Por otro lado, se consideran como variables representativas de las políticas de CTeI orientadas a impulsar la demanda, al personal RENIECYT por millón de habitantes, ya que a partir de 2006 entra en vigor el funcionamiento de dicha institución en el DOF. El RENIECYT forma parte del padrón de empresas y organizaciones que se dedican a la investigación y desarrollo tecnológico, como una variable proxy de las empresas y organizaciones no gubernamentales (ONG) que se dedican a desarrollar tecnología. Los datos de esta variable también fueron tomados de los Diagnósticos Estatales de Ciencia, Tecnología e Innovación 2014. Se asume que a partir del 2002, con el impulso explícito a la incorporación de empresas, esta variable (RENIECYT) mostrará un peso mayor. Adicionalmente, se toman en cuenta las capacidades de las empresas, medidas por las certificaciones ISO e inversión extranjera directa (IED) como variables de control para el desarrollo de capacidades tecnológicas y transferencia de tecnología del extranjero. Con este propósito se toman datos de la Secretaría de Economía para verificar el número de empresas con certificación ISO por entidad federativa. Además se incorpora al modelo, la IED realizada por empresas trasnacionales en México ya que esta inversión supone en una gran cantidad de casos, una transferencia de tecnología.

Como variables adicionales que capturan las prácticas de CTeI, se consideran las matrículas de licenciaturas y posgrados de universidades y tecnológicos, ya que no es igual la matrícula escolar entre entidades federativas, y por ello el impacto del desarrollo de ciencia básica es asimilado de manera distinta en los diferentes Estados. Estos datos provienen de la Asociación Nacional de Universidades e Instituciones de Educación Superior (ANUIES) en sus anuarios estadísticos para el mismo periodo. Por otro lado, se contemplan las externalidades geográficas, mediante variables dummy para cada uno de los estados, en los modelos de efectos fijos y aleatorios.

Como variables de control se toman en cuenta los artículos por investigador, producidos por investigadores del SNI, cuyos datos se encuentran también en los Diagnósticos Estatales de Ciencia, Tecnología e Innovación 2014. Finalmente se toman en cuenta variables de control como

7 Como se comentó en secciones anteriores, las viejas prácticas del modelo de CTeI, consisten en la generación de ciencia básica y/o aplicada a través del otorgamiento de becas y estímulos a la investigación, sin que necesariamente la investigación realizada este relacionada con los problemas y/o contextos específicos de cada región.

Para mayor detalle consultar la pagina http://www.foroconsultivo.org.mx/home/index.php/libros-publicados/diagnosticos-y-analisis-de-cti 8 Las series han sido deflactadas y los datos fueron tomados de Secretaria de Economía. 
el tamaño del estado en términos económicos a partir del producto interno bruto a precios de 2008, y el nivel de población por entidad federativa, ambos datos recabados del INEGI.

Para las variables dependientes, se toman como variables proxy las solicitudes de patentes por millón de habitantes, para aproximar la capacidad inventiva de la población en general. Por otro lado se toma el número de patentes solicitadas por entidad federativa, como una aproximación al desempeño en términos absolutos de los SRI. Se decide no tomar en cuenta a las patentes otorgadas, debido a que existen casos de entidades federativas que no registran patentes otorgadas en años específicos, lo que refleja el rezago que tiene esta variable en el tiempo (Meyer, 2000; Griliches, 1990).

El modelo con el cual se pretende contrastar que las políticas de CTeI han tenido efectos positivos en la capacidad innovadora de los SRI en México, es un modelo de efectos aleatorio. En las primeras secciones de este artículo se ha señalado que existe una relación inobservable en los SRI, donde los temas locales tienen una influencia muy fuerte sobre los resultados innovadores . Tales fenómenos pueden explicar la correlación serial entre el término de error idiosincrásico y el término de perturbación aleatorio. Es por ello que se incluyen variables de control como el tamaño de las entidades medido por el PIB y la población, y la productividad científica por el número de artículos por investigador, por lo cual de manera ex ante se propone un modelo de efectos aleatorios y no un modelo de efectos fijos (Wooldridge, 2010). Los modelos propuestos son los siguientes:

\section{Ecuación 1:}

$$
\begin{gathered}
\text { Pathab }_{i t}=\beta_{0}+\beta_{1}\left(\text { SNI }_{i t}\right)+\beta_{2}\left(\text { Beca }_{i t}\right)+\beta_{3}\left(\text { Reniecyt }_{i t}\right)+\beta_{4}\left(\text { IED }_{i t}\right)+\beta_{5}\left(\text { ISO }_{i t}\right) \\
+\beta_{6}\left(\text { Matricula }_{i t}\right)+\beta_{7}\left(\text { Articulos }_{i t}\right)+\beta_{8}\left(\text { PIB }_{i t}\right)+\beta_{9}\left(\text { poblacion }_{i t}\right)
\end{gathered}
$$

Ecuación 2:

$$
\begin{aligned}
\text { Pat }_{i t} & =\beta_{0}+\beta_{1}\left(\text { SNI }_{i t}\right)+\beta_{2}\left(\text { Beca }_{i t}\right)+\beta_{3}\left(\text { Reniecyt }_{i t}\right)+\beta_{4}\left(\text { IED }_{i t}\right)+\beta_{5}\left(\text { ISO }_{i t}\right) \\
& +\beta_{6}\left(\text { Matricula }_{i t}\right)+\beta_{7}\left(\text { Articulos }_{i t}\right)+\beta_{8}\left(\text { PIB }_{i t}\right)+\beta_{9}\left(\text { poblacion }_{i t}\right)
\end{aligned}
$$

Donde se definen a las variables como:

- Pathab: Patentes solicitadas por millón de habitantes.

- Pat: Patentes solicitadas.

- SNI: Investigadores del sistema nacional de investigadores por millón de habitantes.

- Beca: Becarios por millón de habitantes.

- Reniecyt: Personal Reniecyt por millón de habitantes.

10 Además de efectos como diferencias y proximidades geográficas, factores culturales y políticos, que influyen sobre 
- IED: Inversión extranjera directa en millones de dólares.

- ISO: Número de empresas con certificación ISO.

- Matricula: Es la suma de matrícula de estudiantes de licenciaturas y posgrados.

- Artículos: Número de artículos por investigador del sistema nacional de investigadores.

- PIB: Producto interno bruto a precios constantes de 2008.

- Población: Población en número de personas.

Se espera que las políticas de CTeI tengan un efecto positivo sobre el funcionamiento de los SRI y su capacidad innovadora evaluada a partir de las patentes solicitadas por millón de habitantes y las patentes solicitadas a nivel estatal. Se consideran variables de la vieja política de CTeI a los investigadores del SNI y becarios CONACYT. También se incluyen variables de la nueva política de CTeI como personal Reniecyt, inversión de empresas, y capacidades tecnológicas de empresas, además de variables de control como las matrículas de estudiantes. A partir del modelo se revisará el posible efecto no lineal en la formación de capital humano, específicamente en las matriculas escolares, y el efecto desglosado de investigadores por categoría (SNI_C, SNI_I, SNI_II y SNI_III).

Por último, debido a que los datos contienen un componente de series de tiempo y un componente transversal es que se tiene que realizar una prueba de cointegración para verificar la relación en el largo plazo. El método más común para probar estas relaciones es con la metodología Engle-Granger donde se supone que las variables tienen el mismo orden de integración para después calcular la no existencia de una raíz unitaria en los términos de perturbación. Sin embargo, las pruebas de raíz unitaria difieren con las pruebas estándar de series de tiempo (DF y ADF), en tanto que el panel: i) Permite diferentes grados de heterogeneidad entre los cortes transversales; ii) No se puede estar seguro de rechazar una raíz unitaria; iii) La adición de mayores componentes de corte transversal provee de mayor información comparado con las pruebas ADF en series de tiempo. Por lo anteriormente señalado, se propone emplear los estadísticos LL (prueba de Levin y Lin) donde la hipótesis nula es la existencia de una raíz unitaria, y el estadístico IPS (Im, Pesaran, y Shin), donde adicionalmente la hipótesis nula es la existencia de una raíz unitaria. La principal diferencia entre ambas pruebas es que el primer estadístico no permite una raíz unitaria en los cortes transversales, en tanto que el segundo estadístico verifica esta condición, con la ayuda de estas pruebas es que se corrobora la cointegración en el panel de datos. 


\section{Análisis de resultados.}

En esta sección se muestra una discusión de los principales resultados obtenidos de las dos ecuaciones propuestas. Se describen los resultados de ambas ecuaciones y se les contrasta con la posibilidad de una relación no lineal con las patentes y las patentes por millón de habitantes, y las matrículas escolares. Se observa generalmente una relación no lineal entre las variables de educación y sus rendimientos en la economía, donde las patentes forman parte del cambio tecnológico y del sistema económico en general.

Los resultados de los primeros modelos en el cuadro 5 muestran que todas las ecuaciones deben ser estimadas por el modelo de efectos aleatorios, ya que no es contundente la prueba de Hausman para efectos fijos, y además de ello las pruebas F de significancia global y los estadísticos $\mathrm{R}^{\wedge} 2$, denotan que el modelo es significativo. Esto significa que los SRI deben de tomar en cuenta las diferencias regionales como mecanismo para impulsar el desarrollo de la capacidad innovadora. Estas diferencias se toman a partir de variables dicotómicas englobadas y resumidas en el coeficiente de la constante, lo que sugiere que los efectos locales determinan al funcionamiento de los SRI. Algunas de estas variables que podrían considerarse en futuros modelos son: la consolidación de las entidades encargadas de implementar la política de CTeI en los estados, la infraestructura industrial y educativa. Estas variables más asociadas a la política de largo plazo de la región se incorporan desde el ámbito institucional, fundamental para la operación de los SRI (Nelson y Rosenberg, 1993; Abdel Musik, 2000; Hollingsworth, 2000).

Las ecuaciones muestran también que el gasto realizado en becas de posgrado por parte del CONACYT no es significativo en ninguno de los modelos. Esta situación se mitiga en parte si se considera la variable relativa a las matrículas de licenciatura y posgrado. Estas variables son significativas a nivel individual y existe una relación muy estrecha entre las becas asignadas y las matrículas de los estados. Sin embargo, esto no exime al hecho de que se requiere de una revisión de las viejas prácticas de $\mathrm{CTeI}$ en el caso de las becas, dado que al menos en tiempo presente no dan los resultados esperados en cuanto a la consolidación de un SRI y el desarrollo de capacidades innovadoras. Una explicación alternativa del débil impacto de esta variable podemos encontrarla en la movilidad estudiantil y de investigadores. Los estudiantes de posgrado emigran de su estado originario hacia aquellas localidades en donde se encuentran los posgrados reconocidos por el CONACYT. Esta movilidad inicial puede continuar hacia posgrados en el extranjero por lo que hay una merma de la masa crítica de las regiones (Dutrenit y Ramos, 2012). Esta movilidad interna (en México) y externa (en el extranjero) afecta también la incorporación de capital humano de alto nivel en las empresas y universidades del país. En otros estudios se ha documentado la movilidad de inventores mexicanos que una vez formados con recursos públicos hasta su doctorado se van a trabajar a empresas de otros países, principalmente Estados Unidos (Díaz y Aboites, 2013). 
Cuadro 5

\section{Resultados de los primeros modelos}

\begin{tabular}{|c|c|c|c|c|c|c|c|c|}
\hline \multirow[b]{2}{*}{ Variable } & \multicolumn{2}{|c|}{ Pat } & \multicolumn{2}{|c|}{ Pat } & \multicolumn{2}{|c|}{ Pathab } & \multicolumn{2}{|c|}{ Pathab } \\
\hline & Coeficiente & P-value & Coeficiente & P-value & Coeficiente & P-value & Coeficiente & P-value \\
\hline Constante & 91.954 & 0.224 & 36.878 & 0.709 & -20.687 & 0.270 & 8.649 & 0.706 \\
\hline SNI & 0.066 & 0.022 & 0.059 & 0.052 & 0.014 & 0.064 & 0.018 & 0.017 \\
\hline Beca & 0.010 & 0.541 & 0.011 & 0.519 & 0.006 & 0.138 & 0.006 & 0.183 \\
\hline Reniecyt & 0.152 & 0.033 & 0.161 & 0.027 & 0.041 & 0.025 & 0.037 & 0.045 \\
\hline $\log ($ IED) & -0.364 & 0.307 & -0.359 & 0.315 & -0.119 & 0.206 & -0.120 & 0.202 \\
\hline ISO & 0.219 & 0.001 & 0.213 & 0.001 & 0.036 & 0.001 & 0.039 & 0.001 \\
\hline Matricula & 0.000 & 0.001 & 0.000 & 0.006 & 0.000 & 0.104 & 0.000 & 0.012 \\
\hline Artículos & 2.250 & 0.556 & 3.020 & 0.441 & 1.987 & 0.047 & 1.577 & 0.116 \\
\hline $\log (\mathrm{PIB})$ & 0.093 & 0.984 & 1.263 & 0.795 & 2.116 & 0.067 & 1.479 & 0.194 \\
\hline $\log$ (población) & -8.448 & 0.096 & -4.367 & 0.530 & -0.490 & 0.695 & -2.650 & 0.101 \\
\hline Matricula_raiz & & & -0.117 & 0.378 & & & 0.062 & 0.046 \\
\hline & & & & & & & & \\
\hline Rho & 0.346 & & 0.336 & & 0.389 & & 0.418 & \\
\hline $\mathrm{R}^{\wedge} 2$ & 0.832 & & 0.829 & & 0.608 & & 0.630 & \\
\hline Durbin-Watson & 1.825 & & 1.841 & & 1.694 & & 1.723 & \\
\hline Observaciones & 160 & & 160 & & 160 & & 160 & \\
\hline $\begin{array}{l}\text { Prueba de } \\
\text { Hausman }\end{array}$ & Chi & P-value & Chi & P-value & Chi & P-value & Chi & P-value \\
\hline & 13.190 & 0.154 & 13.456 & 0.199 & 9.906 & 0.358 & 10.501 & 0.398 \\
\hline Prueba F & $\mathbf{F}$ & P-value & $\mathbf{F}$ & P-value & $\mathbf{F}$ & P-value & $\mathbf{F}$ & P-value \\
\hline & 55.565 & 0.001 & 50.385 & 0.001 & 17.435 & 0.001 & 17.613 & 0.001 \\
\hline
\end{tabular}

Se muestran los resultados del modelo, así como los valores po "P-value" para cada coeficiente calculado, ya que es más instructivo ver el nivel de significancia de esta manera. Además se muestran las pruebas de Hausman para efectos fijos, y las pruebas F para significancia global del modelo.

En los modelos propuestos, el Reniecyt tiene mayor peso que la variable relativa a los investigadores del SNI. Se puede conjeturar que el mayor peso del Reniecyt se explica por el cambio de la política de CTeI a partir del 2002 cuando se incentiva la incorporación de las empresas a los programas de financiamiento del CONACYT. Este hallazgo si bien es importante, debe ser tomado con cautela, ya que parte del personal Reniecyt también es mimbro del SNI. Se supone que el diferencial explica la mayor tendencia al patentamiento por un incremento en la actividad innovadora de las empresas en las diferentes entidades federativas. Por otro lado, el impacto en los SRI es más fuerte cuando existen capacidades tecnológicas previas en las empresas, como lo son las certificaciones ISO. Por otra parte, un hallazgo interesante es el efecto de expulsión generado por la IED, ya que la IED está asociada negativamente a la solicitud de patentes (aún y cuando esta variable no resulta significativa). Una posible explicación a este comportamiento es mostrada por Dutrenit (2009), ya que las trasnacionales vienen a México a generar derramas tecnológicas y explotar su propia tecnología, que ya fue patentada en alguno de los países originarios de la trasnacional, y las 
derramas que éstas generan, producen nuevos conocimientos que no necesariamente son patentados, pues generalmente las trasnacionales explotan sus ventajas comparativas, las cuales están basadas en la reducción de costos. Respecto a la variable investigadores SNI se observa que su impacto ocurre solamente cuando se consideran las publicaciones de los investigadores. En este caso se observa un efecto positivo en los SRI y su capacidad innovadora. Tales efectos son significativos en el tercer modelo.

Por último en estos primeros modelos, se observa que en el modelo 4, la variable raíz de la matrícula escolar es significativa y positiva, lo cual se interpreta como la existencia de una relación no lineal entre los SRI y las matrículas escolares. Lo que confirma la postura evolutiva, que señala como principal limitante de los modelos neoclásicos la linealidad en sus conclusiones. Asimismo, tomar en cuenta la raíz cuadrada de una variable denota una forma funcional con rendimientos decrecientes, donde en los valores más pequeños se observa un mayor rendimiento. Es decir, las entidades con poca matrícula tienen un rendimiento mayor sobre su impacto en la solicitud de patentes que aquellos estados en donde hay una matrícula muy grande. Sin embargo, no se debe malentender el concepto, pues la gran cantidad de matrícula de los estados más poblados y desarrollados como el DF, Jalisco, Nuevo León entre otros tienen en términos absolutos un mayor nivel de patentamiento, y su gran matrícula escolar tiene un impacto muy fuerte sobre este patentamiento, esto es, que en volumen de matrícula los estados más poblados generan mucha ciencia básica y/o aplicada la cual es transformada en patentamientos o solicitudes de patentamiento, y aunque el impacto de esta matricula es mayor en estados menos poblados, estos no alcanzan en volumen el nivel de pantentamiento de estados más grandes en términos de matrícula escolar.

Un análisis más detallado que considere las diversas categorías de los investigadores del SNI: candidatos, nivel I, nivel II y nivel III, arroja evidencia más sólida para explorar alternativas explicativas respecto a las políticas de ciencia y tecnología centradas en la oferta frente a aquellas orientadas a impulsar la CTeI y su impacto en los SRI y su capacidad innovadora. Estos modelos se presentan en el cuadro 6 . 
Cuadro 6

Resultados de los modelos con desglose de Investigadores por categoría

\begin{tabular}{|c|c|c|c|c|c|c|c|c|}
\hline & \multicolumn{2}{|c|}{ Pat } & \multicolumn{2}{c|}{ Pat } & \multicolumn{2}{c|}{ Pathab } & \multicolumn{2}{c|}{ Pathab } \\
\hline Variable & Coeficiente & P-value & Coeficiente & P-value & Coeficiente & P-value & Coeficiente & P-value \\
\hline Constante & 77.186 & 0.272 & 72.711 & 0.427 & -18.569 & 0.316 & 11.120 & 0.599 \\
\hline SNI_C & 0.233 & 0.098 & 0.232 & 0.101 & 0.059 & 0.124 & 0.064 & 0.089 \\
\hline SNI_I & -0.075 & 0.237 & -0.074 & 0.243 & 0.020 & 0.244 & 0.018 & 0.283 \\
\hline SNI_II & -0.196 & 0.258 & -0.196 & 0.263 & -0.070 & 0.139 & -0.067 & 0.157 \\
\hline SNI_III & 1.190 & 0.000 & 1.185 & 0.001 & 0.093 & 0.287 & 0.140 & 0.102 \\
\hline Beca & -0.002 & 0.882 & -0.002 & 0.888 & 0.005 & 0.215 & 0.004 & 0.362 \\
\hline Reniecyt & 0.149 & 0.041 & 0.148 & 0.048 & 0.049 & 0.014 & 0.043 & 0.023 \\
\hline Log(IED) & -0.345 & 0.323 & -0.345 & 0.325 & -0.124 & 0.195 & -0.117 & 0.217 \\
\hline ISO & 0.213 & 0.001 & 0.213 & 0.001 & 0.033 & 0.001 & 0.038 & 0.001 \\
\hline Matrícula & 0.001 & 0.001 & 0.000 & 0.068 & 0.001 & 0.266 & 0.001 & 0.014 \\
\hline Artículos & 4.771 & 0.203 & 4.864 & 0.206 & 2.203 & 0.031 & 1.872 & 0.060 \\
\hline Log(PIB) & 0.291 & 0.946 & 0.412 & 0.927 & 1.755 & 0.125 & 1.103 & 0.295 \\
\hline Log(población) & -7.333 & 0.118 & -7.030 & 0.278 & -0.418 & 0.733 & -2.589 & 0.082 \\
\hline Matrícula_raiz & & & -0.009 & 0.942 & & & 0.064 & 0.031 \\
\hline & & & & & & & & \\
\hline Rho & 0.379 & & 0.372 & & 0.409 & & 0.483 & \\
\hline R^2 & 0.859 & & 0.858 & & 0.629 & & 0.669 & \\
\hline Durbin-Watson & 1.954 & & 1.961 & & 1.754 & & 1.757 & \\
\hline Observaciones & 160 & & 160 & & 160 & & 160 & \\
\hline $\begin{array}{c}\text { Prueba de } \\
\text { Hausman }\end{array}$ & Chi & P-value & Chi & P-value & Chi & P-value & Chi & P-value \\
\hline & 12.235 & 0.427 & 12.441 & 0.492 & 9.684 & 0.644 & 13.620 & 0.401 \\
\hline Prueba F & F & P-value & F & P-value & F & P-value & F & P-value \\
\hline & 54.654 & 0.001 & 50.303 & 0.001 & 15.180 & 0.001 & 16.884 & 0.001 \\
\hline
\end{tabular}

Se muestran los resultados del modelo, así como los valores po "P-value" para cada coeficiente calculado, ya que es más instructivo ver el nivel de significancia de esta manera, además se muestran las pruebas de Hausman para efectos fijos, y las pruebas $F$ para significancia global del modelo.

Los resultados de los modelos en el cuadro 6 muestran que todas las ecuaciones deben ser estimadas por el modelo de efectos aleatorios, ya que se no es contundente la prueba de Hausman para efectos fijos. Las pruebas $\mathrm{F}$ de significancia global y los estadísticos $\mathrm{R}^{\wedge} 2$ denotan que el modelo es significativo. Esto implica que los SRI, al igual que en los primeros modelos, deben de tomar en cuenta diferencias regionales, las cuales se toman a partir de variables dicotómicas englobadas y resumidas en el coeficiente de la constante. Estos hallazgos confirman que los efectos locales son muy significativos para explicar el funcionamiento y desempeño de los SRI a través de las capacidades innovadoras regionales. Además de ello, resalta el hecho de que el gasto realizado en becas de posgrado por parte del CONACYT sigue sin ser significativo en ninguno de los modelos. Adicionalmente el signo de este coeficiente es negativo en el primer modelo, lo que denota sus problemas para explicar la capacidad innovadora en las distintas regiones. También en este modelo, 
esta situación se mitiga parcialmente al tomar en cuenta las matrículas de licenciatura y posgrado, ya que estas variables individualmente son significativas y existe una relación muy estrecha entre las becas asignadas y las matrículas de los estados. Sin embargo, la variable de becas de posgrado sigue sin ser relevante para explicar el desempeño en función del patentamiento en las entidades federativas. Además de la movilidad de los becarios (como estudiantes y como graduados), una explicación adicional puede buscarse a partir de los campos de conocimiento en que se otorgan las becas y la concentración de posgrados en los principales estados del país. El resultado es que no se observa un impacto de las becas en la consolidación de los SRI en México y en la promoción de la capacidad innovadora.

El análisis de las variables relativas a los modelos de políticas centrados en la oferta de conocimientos (viejos) y los orientados a impulsar la demanda (CTeI), se observa que la variable relativa al número de partiticpantes en el Reniecyt aunque tiene un impacto, no es mayor al que tienen los investigadores del SNI. A diferencia de los primeros modelos, cuando se realiza un comparativo de las viejas y nuevas políticas de CTeI, se obtiene como conclusión que el impacto del personal Reniecyt ya no es mayor al impacto generado por los investigadores del sistema nacional de investigadores. Particularmente el efecto es mayor si el análisis parte de la diferenciación de niveles en el SNI. Los investigadores nivel III tienen un peso muy alto para explicar el patentamiento, seguidos de los candidatos a investigador nacional, lo que es consistente con otros estudios como (Díaz, 2014; Díaz y Alarcón, 2015). Este resultado coincide con investigaciones previas en dos aspectos: (i) la participación de investigadores consolidados, de alto nivel, de mayor liderazgo, con publicaciones de alto impacto y con acceso a financiamiento externo, son los que registran el mayor número de patentes solicitadas y otorgadas (Díaz y Alarcón, 2014; Díaz y Aboites, 2013; Huang, Feeney y Welch, 2011; Huelsbeck y Lehmann, 2006). (ii) la participación de estudiantes e investigadores recién doctorados está asociada en investigaciones previas a una alta propensión a patentar debido a las nuevas ideas y energía que incorporan los investigadores jóvenes (Díaz y Alarcón, 2015; Aboites y Díaz, 2015; Gurmu, Black y Stephan, 2010).

Asimismo se observa que las capacidades tecnológicas, medidas en relación a las certificaciones ISO tienen un impacto significativo en el desempeño del SRI, cuando se mide a partir de las patentes solicitadas. Estudios previos muestran también que la certificación les permite a las empresas equiparar sus procesos y convertirse en exportadoras o articularse a cadenas de exportación como proveedoras de multinacionales (Abdel Musik, 2000).

Como en los modelos previos, la IED tiene una relación negativa con la propensión a patentar, además de que no resulta ser una variable significativa para explicar el desempeño de los SRI y su capacidad innovadora. En los países industrializados la IED generalmente está asociada positivamente al incremento de la propiedad intelectual, esto implica que ese tipo de inversión se 
encuentra estrechamente vinculada a los flujos de conocimiento. Se puede conjeturar que la IED que llega a México es inversión que no está asociada al conocimiento. Lo anterior sugiere que es una inversión que busca aprovechar dos características esenciales de la economía mexicana: la energía (petrolera) y por el otro, la mano de obra barata principalmente a través de la industria maquiladora de exportación (IME). Sin embargo, si observamos el registro de patentes en el IMPI, a partir del TLCAN, encontramos que el crecimiento de patentes extranjeras es muy dinámico en comparación a lo que sucedía antes del TLCAN (Aboites y Soria, 2008). Este hallazgo tendrá que estudiarse con mayor detalle en futuras investigaciones.

En favor de los investigadores SNI, se puede señalar que su impacto es más fuerte si se considera que sus publicaciones en artículos generan efectos positivos sobre los SRI y que estos efectos son significativos en el tercer y cuarto modelo del cuadro 6. Esto se debe en alguna medida, a las derramas de tecnología generadas por los investigadores miembros del SNI, pues sus publicaciones son importantes para generar nuevas ideas y/o conocimiento que puede ser patentado, sobre todo en las áreas de ingeniería y ciencias afines.

Por último en los modelos presentados en el cuadro 6, se observa que la variable raíz de la matrícula escolar es significativa y positiva en el cuarto caso, lo cual se interpreta como la existencia de una relación no lineal entre los SRI, su capacidad innovadora y las matrículas escolares. Lo que confirma la postura evolutiva que señala como principal limitante de los modelos neoclásicos la linealidad en sus conclusiones. Esto lleva a señalar que no es necesariamente la existencia de una gran matrícula escolar la que impacta en los SRI, sino que es tal vez la focalización de estas matrículas en áreas como la Ingeniería y ciencias afines. Hay que considerar también que la raíz cuadrada de una variable, denota una forma funcional con rendimientos decrecientes, donde en los valores más pequeños se observa un mayor rendimiento. Es decir, las entidades con poca matrícula tienen un rendimiento mayor sobre su impacto en la solicitud de patentes que aquellos estados en donde hay una matrícula muy grande, lo que significa que en estados con poca matrícula existen más incentivos a orientar la investigación de posgrado y licenciatura hacia el patentamiento. Tomando en cuenta la relación en el largo plazo, la prueba de cointegración LL muestra los siguientes resultados, en el cuadro 7. 
Cuadro 7

\section{Resultados de la prueba LL para las variables del modelo}

\begin{tabular}{|c|c|c|}
\hline Variable & LL & Nivel de la prueba \\
\hline SNI & $-48.805^{* *}$ & un rezago \\
\hline Reniecyt & $-1.362^{*}$ & un rezago \\
\hline IED & $-15.574^{* * *}$ & un rezago \\
\hline PIB & $-16.861^{* * *}$ & un rezago \\
\hline Población & $-14.070^{* * *}$ & un rezago \\
\hline Matrícula & $-14.995^{* * *}$ & un rezago \\
\hline Artículos & $-150.001^{* * *}$ & un rezago \\
\hline ISO & $-1.361^{*}$ & un rezago \\
\hline Beca & $-9.984^{* * *}$ & un rezago \\
\hline & & \\
\hline
\end{tabular}

Los datos de la tabla, muestran el estadístico LL con ***, ** $y *$, que muestran los niveles de significancia al $1 \%, 5 \%$ y $10 \%$.

Se observa en los resultados del cuadro 7 que todas las variables pasan la prueba de raíz unitaria con un rezago, por lo que los datos no son concluyentes para la existencia de una raíz unitaria en los residuos, y por ello permiten realizar la prueba de cointegración para ambos modelos.

Cuadro 8

\section{Resultados de la prueba LL para cointegración}

\begin{tabular}{|c|c|c|}
\hline Ecuación & LL & Nivel de la prueba \\
\hline Pathab & $-3.582^{* * *}$ & En nivel \\
\hline Pat & $-204.473^{* * *}$ & En nivel \\
\hline & & \\
\hline
\end{tabular}

Los datos de la tabla, muestran el estadístico LL con ***, ** y*, que muestran los niveles de significancia al $1 \%, 5 \%$ y $10 \%$.

Los datos del cuadro 8 permiten señalar que existe una cointegración, y con ello una relación que se sostiene en el largo plazo, esto es, que las viejas y nuevas políticas de CTeI, en realidad han impactado a los SRI y su capacidad innovadora, por lo menos en el periodo señalado (2007-2011). 


\section{$\rightarrow$ 6. Conclusiones.}

Se ha evaluado el impacto que la política de CTeI tiene en los SRI y su capacidad innovadora en el periodo 2007 a 2011. Se articulan dos modelos. En el primero se analizan variables relativas a la política tradicional de ciencia y tecnología centrada en la oferta de conocimiento, a partir de variables como el número de becas de posgrado del CONACYT e investigadores del SNI. En el segundo modelo se exploran variables que dan cuenta de una política orientada a promover la innovación y la participación de las empresas a partir de los nuevos programas de financiamiento del CONACYT. Se analizan variables como capacidades tecnológicas, número de inscritos en el Reniecyt, la IED, entre otras.

En el análisis se debate la pertinencia de usar a las patentes como indicador del desempeño de los SRI y su capacidad innovadora. La discusión enfatiza la parcialidad del indicador pero, por otro lado, se señala la accesibilidad y confiabilidad de la información de patentamiento a nivel estatal. Esta discusión se enmarca en las propuestas de la economía evolutiva que analiza la relación no lineal entre las variables de política de CTeI y el desempeño de los SRI a través de su capacidad innovadora. Los SRI se equiparan a las 32 entidades federativas del país considerando la disponibilidad de la información, mediante la implementación de un análisis de regresión con datos en panel y con efectos aleatorios.

La implementación de esta regresión ha dado como resultado que los efectos aleatorios son significativos, lo que representa la existencia de una relación inobservable en los SRI. Los temas locales tienen una influencia muy fuerte sobre la capacidad innovadora, y la interdependencia en tiempos pasados y presentes juega un rol significativo, lo cual de alguna manera determina una correlación serial entre el término de error idiosincrásico y el término de perturbación aleatorio. Esto significa que las políticas para impulsar el desarrollo de los SRI deben de tomar en cuenta las diferencias regionales, las cuales se describen a partir de variables dicotómicas englobadas y resumidas en el coeficiente de la constante, lo que confirma que los efectos locales determinan al funcionamiento de los SRI y por tanto la capacidad innovadora. Los hallazgos más significativos son: el escaso impacto de las becas de posgrado en las regiones, y la relación negativa de la IED con el número de patentes solicitadas. Respecto a los hallazgos esperados se confirma por ejemplo que los investigadores del SNI nivel 3 y nivel candidato tienen un impacto importante en la capacidad innovadora de las diferentes regiones del país, así como la matrícula en licenciatura y posgrado y las capacidades tecnológicas medidas a través del número de empresas con certificación ISO. Sin duda, esta línea de análisis genera más interrogantes de las que responde pero permite vislumbrar algunos elementos significativos para el diseño de políticas de CTeI y sobre todo para explorar a través de otras variables el desempeño del sistema y la capacidad innovadora de las regiones. 
Esta relación ha sido comprobada en el largo plazo, ya que los estadísticos LL para los términos de error muestran que existe una cointegración, y con ello una relación que se sostiene en el largo plazo. Esto es, que las viejas y las nuevas políticas de CTeI en realidad han impactado a los SRI, por lo menos en el periodo señalado (2007-2011), donde se establece que son políticas asociadas a la capacidad innovadora de los estados.

Limitaciones importantes de la investigación hacen referencia a la variable proxy utilizada para evaluar la capacidad innovadora como medida del desempeño de los SRI, ya que las patentes como se ha señalado, no concretan todas las invenciones protegidas en productos $\mathrm{y} / \mathrm{o}$ procesos exitosos en el mercado. Sin embargo, son una medida del esfuerzo dedicado a esa actividad en recursos humanos y financieros, infraestructura, entre otros orientada a desarrollar innovaciones. En cuanto al alcance de esta investigación, aunque se sabe que el gasto en $\mathrm{I}+\mathrm{D}$ es una de las variables más importantes en la generación de innovaciones, no existe un desglose del gasto gubernamental en I+D por entidad federativa que pueda ser utilizado para estos fines. Es por ello que solo se puede aproximar mediante el gasto becas en CONACYT, miembros SNI y personal Reniecyt. Futuras líneas de investigación deberán centrar sus esfuerzos en abordar el tema de cómo mejorar el bienestar de la población con un mejor desempeño de los SRI, y de cuánto contribuye la capacidad innovadora generada en los SRI a la economía tanto nacional como regional, realizando comparativos de grupos de entidades federativas con desempeños diferentes y/o similares en sus políticas y resultados en $\mathrm{I}+\mathrm{D}$. 


\section{Bibliografía y referencias documentales}

Abdel Musik, G. (2000). "Regional and local systems of innovation in Aguascalientes", en Mario Cimoli (ed.), Developing innovation systems. Mexico in a global context, Londres, Continuum.

Aboites, J. y C. Díaz (2015). "Introducción”, en Jaime Aboites y Claudia Díaz (coords.), Inventores y patentes académicas: La experiencia de la UAM, México, UAM/ Siglo XXI editores.

Aboites, Jaime y Manuel Soria (2008). Economía del Conocimiento y Propiedad Intelectual: Lecciones para la Economía Mexicana. México, UAM-X y Siglo XXI.

Alarcón, Moisés y Díaz, Claudia (2014). "The technology based sectors in Mexico: An analysis for the firm size and the production scale", en Economic Review of Galicia, vol. 23, núm. 4, pp. 49-60.

Asheim, B., y Gertler, M. (2005). “The Geography of Innovation: Regional Innovation Systems”, en J. Fagerberg, D. C. Mowery y R. R. Nelson (editors), The Oxford Handbook of Innovation, Oxford, University Press.

Asheim, B. T. (1998). "Localized innovation networks in a global economy: A comparative analysis of endogenous and exogenous regional development approaches", en Comparative Social Research, vol. 17, pp. 199-240.

Balzat, M., \& Hanusch, H. (2004). "Recent trends in the research on national innovation systems", en Journal of Evolutionary Economics, vol. 14, núm. 2, pp. 197-210.

Basile, R., Capello, R., y Caragliu, A. (2012). "Technological interdependence and regional growth in Europe: Proximity and synergy in knowledge spillovers", en Papers in Regional Science, vol. 91, núm. 4, pp. 697-722.

Boscherini, F., y Yoguel, G. (1996). "La capacidad innovadora y el fortalecimiento de la competitividad de las firmas: el caso de las PYMEs exportadoras argentinas", en Documento de trabajo No. 71, Buenos Aires, CEPAL.

Brancati, R., Frinolli, A., Mastrostefano, V., y Silvani, A. (2000). "Regional Technological Development: some lessons from evaluation activities", en Fourth European Conference on Evaluation of the Structural Funds, Edinburgo.

Casas, Rosalba. (2001). La formación de redes de conocimiento: una perspectiva desde México. México: Anthropos, UNAM-Instituto de Investigaciones Sociales, México.

Corona, J., Dutrenit, G., Puchet, M. y Santiago, F. (2013). "La Co-evolución de las políticas de CTI, el sistema de innovación y el entorno institucional en México", en Gabriela Dutrénit y Zuñiga (coords.), Políticas de eficiencia, tecnología e innovación para el desarrollo. La experiencia latinoamericana, México, FCCyT.

Corona, J., Dutrenit, G., Torres, A. y Vera-Cruz, A. (2011). "Políticas de ciencia y tecnología para el crecimiento y desarrollo económicos propuestas para un desarrollo nacional basado en la innovación", en Federico Novelo (coord.), La UAM ante la sucesión presidencial propuestas de política económica y social para el nuevo gobierno, México, UAM, MC editores.

Cotgreave, P., y Davies, R. (2005). "How can we measure the success of national science policies in the short or medium term?”, en European Journal of Education, vol. 40, núm. 4, pp. 393-404.

Crescenzi, R. (2005). "Innovation and regional growth in the enlarged Europe: the role of local innovative capabilities, peripherality, and education”, en Growth and Change, vol. 36, núm. 4, pp. 471-507. 
Díaz, C. (2002). "Cambio y permanencia: Los requisitos de la transformación. Análisis comparativo de las políticas tecnológicas y el desempeño institucional en México y Corea del Sur”, en Adrián Acosta (coord.), Cambio Institucional, Guadalajara, Universidad de Guadalajara.

Díaz, C. (2007). Los centros de investigación y desarrollo tecnológico en México: regulaciones institucionales y estrategias organizacionales, Guadalajara, Universidad de Gudalajara.

Díaz, C., A. Alarcón y A. Ayala (2011). "Clustering and Innovation Capabilities in the Mexican Software Industry”, en Engineering Management Journal, vol. 23, núm. 4, pp. 47-56.

Díaz, C. (2013), "Ciencia y Tecnología”, en Jalisco a Futuro, Guadalajara, Universidad de Guadalajara y Gobierno del estado de Jalisco.

Díaz, C. (2014). "Patentes académicas en México", en Colección Temas de hoy en la educación superior, México, ANUIES.

Díaz, C. y Alarcón, M. (2015). "Factors related to academic patenting in a Mexican University", en Portland International Conference 2015 Management of the Technology Age, San José, California, IEEE, pp. 1078-1087.

Díaz, C. y J. Aboites (2013). "Institutional and organizational factors associated to academic patenting in a Mexican University: teams' trajectories, networks and performance", en Technology Management in the IT-Driven Services (PICMET), 2013 Proceedings of PICMET'13, Vancouver, IEEE, pp. 10321043.

Diessler, Gabriela (2010). "Las patentes como fuente de información para la innovación en entornos competitivos", en Información, Cultura y Sociedad, vol. 22, pp. 43-77.

Donovan, C. (2007). “The qualitative future of research evaluation”, en Science and Public Policy, vol. 34, núm. 8, pp. 585-597.

Dutrénit, Gabriela (Coord.) (2009). Sistemas regionales de innovación: un espacio para el desarrollo de las pymes el caso de la industria de maquinados industriales, México, Editorial textual, Universidad Autónoma Metropolitana.

Dutrénit, G. y Ramos, C. (2012). "Towards building critical mass of science, technology and innovation: the Mexican experience in policy making", en H. Lastres, C. Pietrobelli, R. Caporali, M. Soares \& M. Matos (coords.), A nova geração de políticas de desenvolvimento produtivo sustentabilidade social e ambiental, Brasilia.

Dutrénit, Gabriela y Zuñiga, Patricia (2013). Políticas de ciencia, tecnología e innovación para el desarrollo La experiencia latinoamericana, México, Foro Consultivo Científico y Tecnológico AC.

Edquist, Charles (1997). Systems of Innovation, London, Cassel.

Edquist, Charles y Hommen, Leif (1999). "Systems of innovation: theory and policy for the demand side", en Technology In Society, vol. 21, pp. 63-79.

Edquist, Charles (2006). "Systems of Innovation: Perspectives and Challenges", en J. Fagerberg, D. C. Mowery Y R. R. Nelson (Eds.), The Oxford Handbook Of Innovation, New York, Oxford University Press.

Edwards, K. y Gordon, T. (1984). "Characterization of Innovations Introduced on the U.S. Market in 1982”, en Report Prepared for the U.S. Small Business Administration, Washington, DC, The Futures Group. 
Feller, I. (2007). "Mapping the frontiers of evaluation of public-sector R\&D programs", Science and Public Policy, vol. 34, núm. 10, pp. 681-690.

Freeman, Christopher (1987). Technology Policy and Economic Performance: Lessons from Japan, Londres, Pinter Publishers.

Furman, J. L., Porter, M. E., y Stern, S. (2002). "The determinants of national innovative capacity", en Research policy, vol. 31, núm. 6, pp. 899-933.

Geels, Frank (2014). "Reconceptualising the co-evolution of firms-in-industries and their environments: Developing an inter-disciplinary Triple Embeddedness Framework", en Research Policy, vol. 43, núm. 2, pp. 261-277.

Godin, B. (2012). “Innovation Studies”: The Invention of a Specialty, en Minerva, vol. 50, núm. 4, pp. 397 421.

Griliches, Z. (1990). "Patent Statistics as Economic Indicators: A Survey”, en Journal of Economic Literature, vol. XXVIII, pp. 1661-1707.

Griliches, Z. (1994). "Productivity, R\&D, and the Data Constraint”, en American Economic Review, vol. 84, núm. 1, pp. 1-23.

Gurmu, S., Black, G. C. y Stephan, P. (2010). “The Knowledge Production Function for University Patenting”, en Economic Inquiry, vol. 48, núm. 1, pp. 192-213.

Hage, J., Jordan, G., y Mote, J. (2007). "A theory-based innovation systems framework for evaluating diverse portfolios of research, part two: Macro indicators and policy interventions", en Science and Public Policy, vol. 34, núm. 10, pp. 731-741.

Hollingsworth, J. R. (2000). "Doing institutional analysis: Implications for the study of innovations", en Review of Political Economy, vol. 7, núm. 4, pp. 595-644.

Huang, W.L., Fenney, M., y Welch, E. (2011). "Organizational and individual determinants of patent production of academic scientists and engineers in the United States", en Science and Public Policy, vol. 38, núm. 6, pp. 463-479.

Huelsbeck, M., \& Lehmann, E. (2007). "German university patenting and licensing: Legally prescribed incentives and institutional determinants of university-industry-technology-transfer", en DRUIDDIME Academy Winter Conference.

Ibrahim, A. R., Zolait, A. H. S., Subramanian, S., y Ashtiani, A. V. (2009). "Organizational innovative capabilities: an empirical study of Malaysian firms", en Journal of Innovation and Business Best Practices, vol. 1, núm. 2, pp. 9-18.

Ivanova, I. y Leydesdorff, L. (2014). "Rotational symmetry and the transformation of innovation systems in a Triple Helix of university-industry-government relations", en Technological Forecasting \& Social Change, vol. 86, pp.143-156.

Leyva, S. L., y Barraza, L. A. S. (2007). "Un análisis de la política de ciencia y tecnología en México (20012006)”, en Estudios Sociales, vol. 16, núm. 30, pp.136-165.

Lucas, R. (1988). "On the mechanics of economic development”, en Journal of Monetary Economics, vol. XXII, pp. 3-42.

Lundvall, B. A. (1992). National systems of innovation, London, Pinter. 
Lundvall, B.A. y S. Borrás (2006). "Science, Technology and Innovation Policy", en J. Fagerberg, D. C. Mowery Y R. R. Nelson (Eds.), The Oxford Handbook Of Innovation, New York, Oxford University Press, 599-631.

Martin, B. R. (2012). "The evolution of science policy and innovation studies", en Research Policy, vol. 41, núm. 7, pp. 1219-1239.

Melkers, J. (2004). "Assessing the outcomes of state science and technology organizations", en Economic Development Quarterly, vol. 18, núm. 2, pp.186-201.

Meyer, Martin (2000). "Does science push technology? Patents citing scientific literature", en Research Policy, vol. 29, núm. 3, pp. 409-434.

Molas-Gallart, J., y Davies, A. (2006). "Toward theory-led evaluation the experience of European science, technology, and Innovation policies", en American Journal of Evaluation, vol. 27, núm. 1, pp. 6482.

Mote, J., Jordan, G., y Hage, J. (2007). "Measuring radical innovation in real time", en International Journal of Technology, Policy and Management, vol. 7, núm. 4, pp. 355-377.

Mora, H. y Soria, M. (2012). "Modalidades de innovación y construcción de capacidad tecnológica endógena de economías en desarrollo: los catalizadores en Brasil, Colombia y México, 1955-2009”, en Economía: Teoría y Práctica, núm. 37, pp. 145-175.

Nelson, R. (1993). National Innovation Systems: A Comparative Study, New York, Oxford University Press.

Nelson, R. y Rosenberg, N. (1993). “Technical Innovation \& National Systems”, en R. R. Nelson (Ed.), Innovation Systems. A Comparative Analysis. New York: Oxford University Press.

Nelson, R.; Winter, S. y Schuette, H. (1976). "Technical Change in an Evolutionary Model”, en Quarterly Journal of Economics, pp. 90-118.

Neuman, M., Camblong, J., Modai, E., Nicolini, J., Fernández, M., y Abrevaya, C. (2015). "Factores que contribuyen al desarrollo de nuevos productos", en Ingenium, vol. 2, núm. 3, pp. 32-29.

Ospina, A. G. (2010). La generación de conocimiento organizacional como factor clave en el desarrollo de la capacidad de innovación. Líneas para el Debate, núm 43, pp. 6-20.

Pavitt, K. (1998). "Do patents reflect the useful research output of universities?", en Research Evaluation, vol. 7, núm. 2, pp. 105-111.

Pavitt, K., Robson, M. y Townsend, J. (1987). "The Size Distribution of Innovating Firms in the UK: 19451983”, en The Journal of Industrial Economics, vol. XXXV, núm. 3, pp. 297-316.

Romer, Paul (1989). “Endogenous Technological Change”, en NBER working paper series No. 3210.

Santiago, Fernando y Natera, José (2014). Tiempos de respuestas de la dinámica económica asociados a la inversión en ciencia, tecnología e innovación en México, México, Foro Consultivo Científico y Tecnológico.

Sarukhán, J. (1989). "The Status of Mexican Science and Technology Research: Potential Avenues for Collaborative Programmes with the United States", en Mexican Studies/Estudios Mexicanos, vol. 5, núm. 2, pp. 265-280. 
Schmookler, Jacob (1951). "Invention and economic development", Unpublished PhD dissertation, U. of Pennsylvania.

Schmookler, Jacob (1952). "The Changing Efficiency of the American Economy: 1869-1938”, en Review of Economics and Statistics, vol. 34, núm. 3, pp. 214-321.

Solow, R. (1957). "Technical change and the aggregate production function", en Review of Economics and Statistics, vol. 39, núm. 3, pp. 312-320.

Soria L., M. (2015), “Conocimiento tecnológico patentado por la UAM 1984-2011”, en J. Aboites y C. Díaz, Inventores y patentes académicas. La experiencia de la UAM, México, Siglo XXI/UAM.

Varela, Gonzalo (2002). "Sistemas de innovación: una comparación entre Japón y México”, en Foro Internacional, vol. XLII, núm. 1, pp. 154-185.

Wooldridge, J.M. (2010). Introductory Econometrics, USA, Cengage Learning, 4th Edition.

Zawislak, P. A., Cherubini Alves, A., Tello-Gamarra, J., Barbieux, D., y Reichert, F. M. (2012). "Innovation capability: from technology development to transaction capability", en Journal of Technology Management \& Innovation, vol. 7, núm. 2, pp. 14-27. 
Números anteriores:

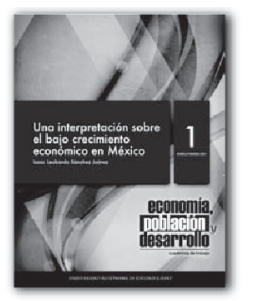

Economía, población y desarrollo.
Cuadernos de trabajo №1

Enero-Febrero 2011
Una interpretación sobre el bajo

crecimiento economico en México
Isaac Leobardo Sánchez Juárez

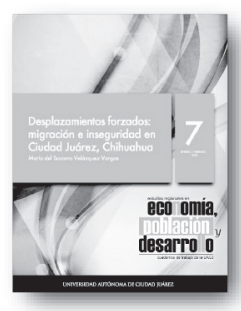

Economia, población y desarroll

Cuadernos de trabajo $\mathrm{N}$ -
Encro-Fcbrcro 2012

Desplazamientos forzados
migración e inseguridad

Ciudad Juárez, Chihuahua
Maria del Socorro Velázquez Varga

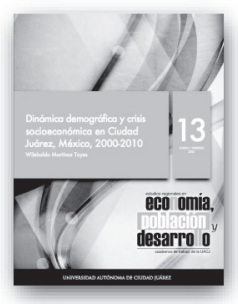

Economiá, población y desarrollo

de trabajo $N$

Dinámica demográ́fica y crisis
socieconómica en Ciudad Juáre Mexico, 2000-2010

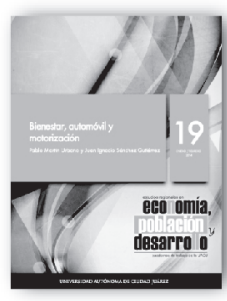

Economía, población y desarroll Enero - Febrero 2014 Bienestar, automóvil y motorización
Pablo Martín Urbano y Miosánchez Gutiérrez

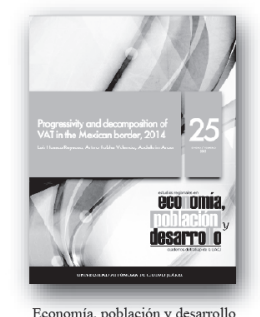

Cuadernos de trabajo No $_{0}$
Conomia,

Encro - Febrero 2015

Progressivity and decomposition of
VAT in the Mexican border, 2014 Abdelkim Araar

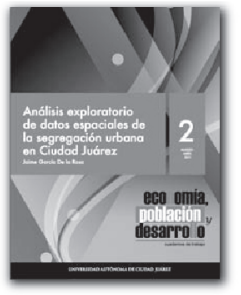

Economía, población y desarrollo.
Cuadernos de trabajo № 2

Marzo-Abril 2011

espaciales de la segregacón

Jaime Garcia De la Rosa

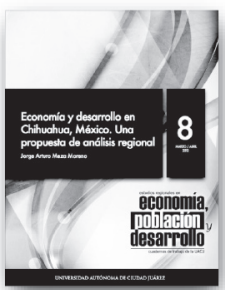

Economia, población y desarrollo

Cuadernos de trabajo №7

Economía y desarrollo en

propuesta de análisis regiona

Jorge Arturo Meza Moreno

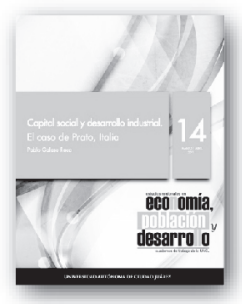

Economía, población y desarrollo

Marzo - Abri1 2013

Capital social y desarrollo

Pablo Galaso Reca

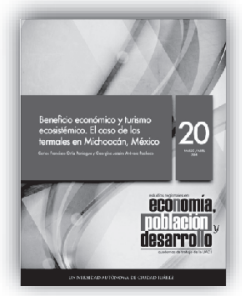

Economia, población y desarrollo

Marzo - Abril 2014

Beneficio económico y turismo

enico. El caso de las ter
en Michoacán, Mésico

arlos Franciseo Ortiz Paniagu

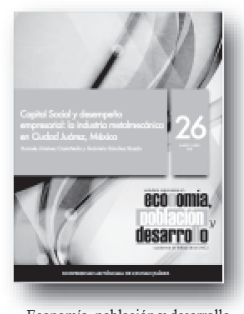

Economía, población y desarrollo

Cuadernos de trabajo № 26
Marzo - Abril 2015

Capital Social y desempeño empresa
la industria metalmecánica en

a industria metalmecánica en
Ciudad Juárez, México

Ramsés Jiménez Castañeda y
Gabriela Sáncez Bazán
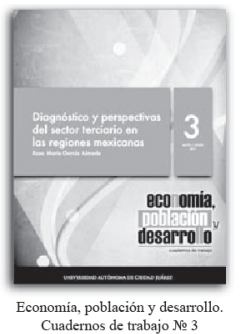

Diagnóstico y perspectivivas

regiones mexicanas
rosa Mária Garcia Almad

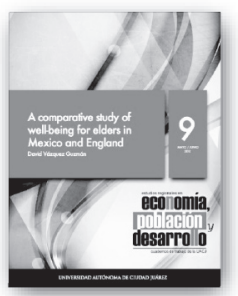

Economia, población y desarrollo

Cuadernos de trabajo № 9
Mayo - Junio 2012

A comparative study of

Mexico and England
David Vázquez Guzmán

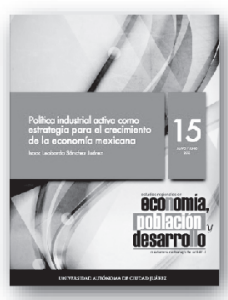

Economia, población y desarroll

Madernos de trabajo
Mayo - Junio 2013

Politica ind ustrial activa como

strategia para el crecimiento

Isaac Lcobardo Ś́nexicana
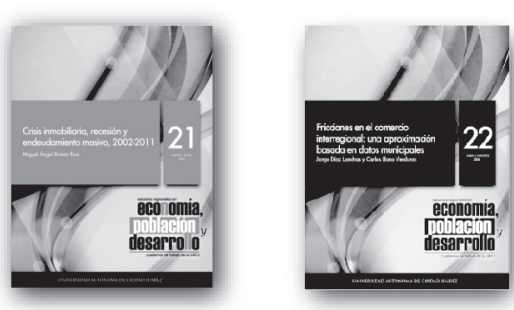

Economia, población y desarrollo

Cuademos de trabajo
Mayo - Junio 2014

Crisis inmobiliaria, recesión y
adeudamiento masivo, $2002-2011$

Miguel Ángel Rivera Rios

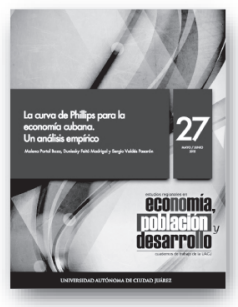

Economia, población y desarrollo

Cuadernos de trabajo № 27

a curva de Phillips para

economía cubana.

Malena Portal Boza, Duniesky Feitó

Economía, población y desarrollo.
Cuadernos de trabsio

julio-Agosto 201

Los indices IDH y FGT en la

mera década del siglo XX

Economía, población y decarrollo

Cuadernos de trabajo №
Julio - Agosto 2012

Political competition and the

edistribution in a federation

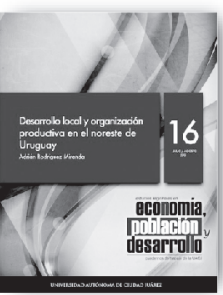

Economía, población y desarrollo

Cuadernos de trabajo №
Julio - Agosto 2013

Desarrollo local y organización
productiva en el noroeste de Urugu

Economía, población y desarrollo

Julio - Agosto 2014

Ficciones en el comercio

basada en datos municipales
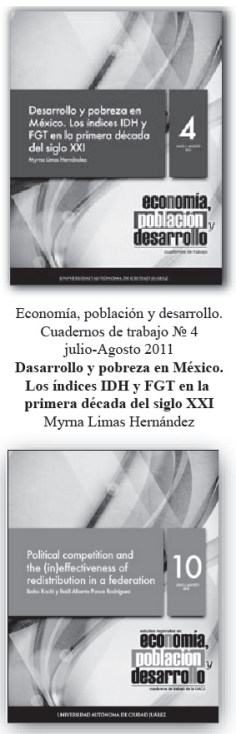

Jorge Diaz Lanchas y Carlos Llano Verduras
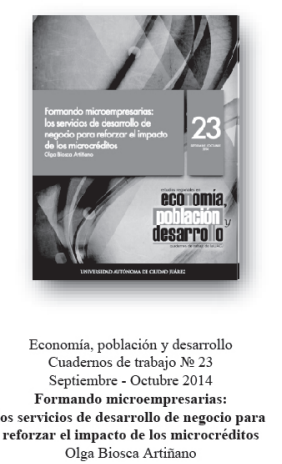

Cuadernos de trabajo № 24

El crecimiento de las regiones

el paradigma del desarrollo

divergente. Un marco térico
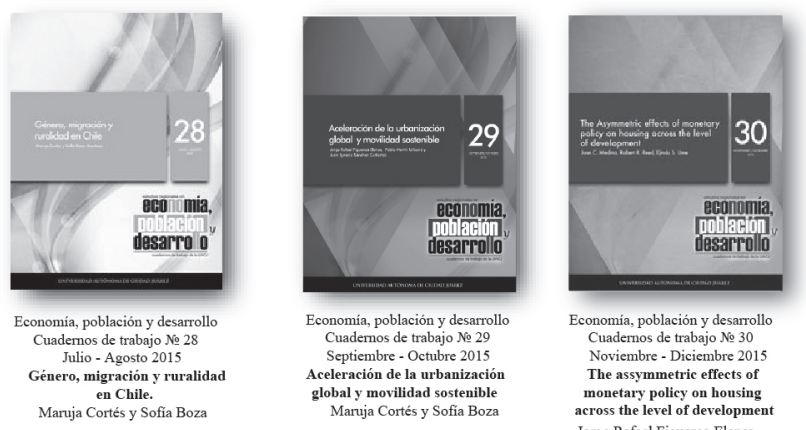

conomía, población y desarrol

Cuadernos de trabajo № 30

The assymmetric effects of

monetary policy on housing

Jorge Rafael Figueroa Elenes,

Juan Ignacio Sánchez Gutiérrez 
Números anteriores:
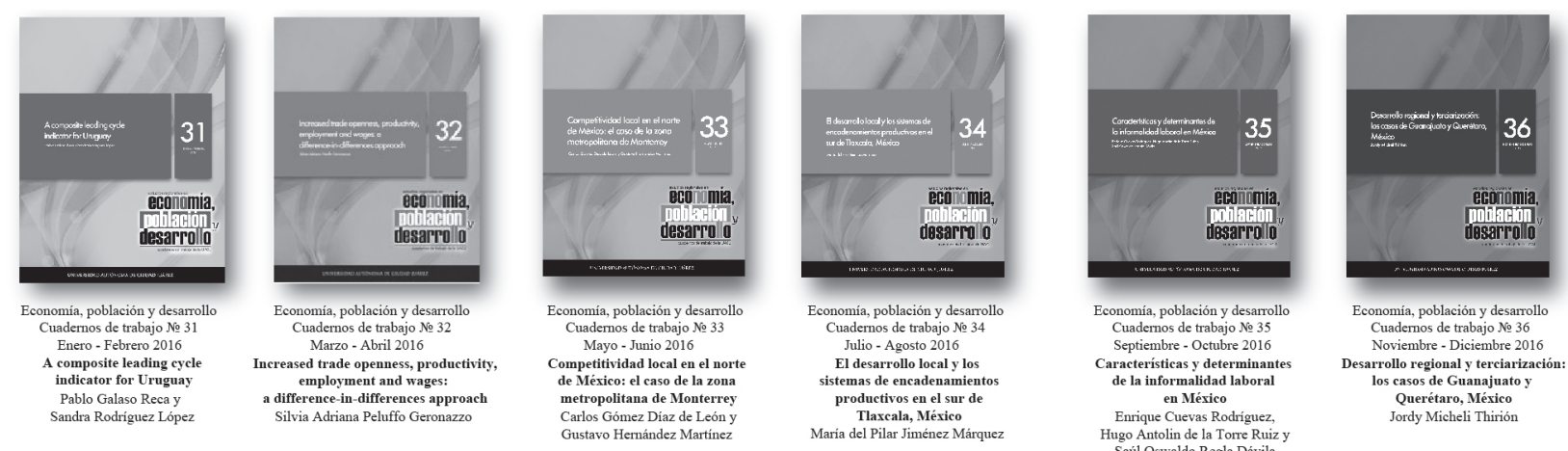

Economía, población y desarrollo Septiembre - Octubre 2016

Caracteristicas $y$ determinantes
de la informalidad laboral en México

Enrique Cucvas Rodrígucz,
Hugo Antolin de lo Torre Ruz

Gustayo Herrández Martinez

Maria del Pilar Jiménez Márquez
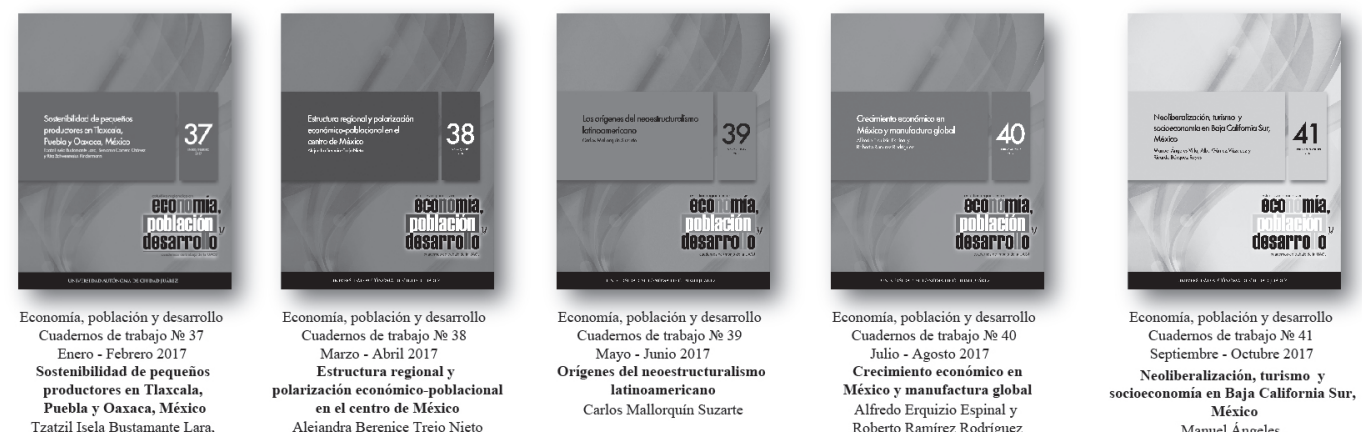

Economia, población y desarrollo Noviembre - Diciembre 2016 Desarrollo regional y terciarización:
los casos de Guanajuato y Querétaro, México

Cuadernos de trabajo № 39 Mayo - Junio 2017

Enero - Febrero 2017 ostenibilidad de pequeños

productores en Tlascala,

zatzil Iscla Bustamante Lara, Benjamin Carrera Chávez y
Rita Schwentesius Rindermanı

Alcjandra Bereniec Trajo Nie

latinoamericano

Economía, población y desarroll
Cuademos de trabajo № 40

Julio - Agosto 2017

Crecimiento economico en
Mésico y manufactura global

Alfredo Erquizio Espinal y

Economía, población y desarrollo

Septiembre - Octubre 2017

Neoliberalización, turismo y
ioeconomía en Baja California

México

Manuel Ángeles,

Ricardo Bórquez

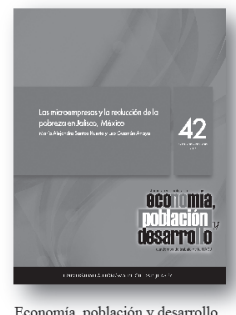

conomía, población y desarrollo Cuadernos de trabajo № 42
Noviembre - Diciembre 2017 Las microempresas y la reducción
de la pobreza en Jalisco, México Maria Alcjandra Santos Hucrta Leo Guzmann Anaya

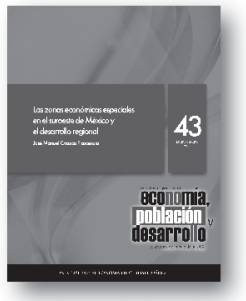

Economia, población y desarrol Cuadernos de trabajo $\mathrm{No}$
Enero - Febrero 2018

Las zonas económicas especiales el desarrollo regional José Manuel Orozco Plascenci

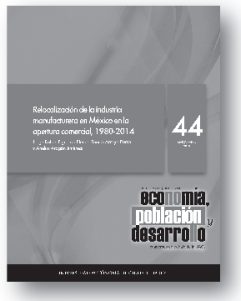

Economia, poblacion y desarrollo Cuadernos de trabajo №
Marzo - Abril 2018

Relocalización de la industria apertura comercial 1980-2014 Jorge Rafael Figueroa Elenes, Tomás Aroyo Parra y
Aneliss Aragón Jiménez

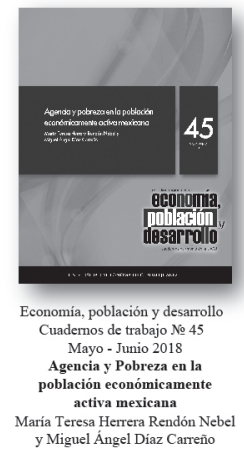




\section{$\rightarrow$ Normas Editoriales}

\section{Para el documento general:}

Tipo de letra: Times New Roman.

Tamaño: 11 puntos.

Interlineado: 1.5 espacios.

Títulos y subtítulos:

El texto principal en 11 puntos. Títulos 12 puntos (en resaltado). Subtítulos 11 puntos. Cada título y subtítulo deberá numerarse bajo el siguiente orden: $1,1.1,2,2.1,2.2 \ldots$

La extensión máxima de los cuadernos de trabajo será de 40 cuartillas.

La primera vez que se emplee una sigla en el texto se especificará primero su equivalencia completa y después la sigla.

\section{Hoja de presentación:}

Título:

14 puntos, centrado, resaltado.

Nombre de autor(es):

12 puntos

Resumen y abstract:

Debe incluir resumen en español y abstract (diez puntos), no mayor a 250 palabras

Palabras clave:

Incluir entre tres y cinco palabras clave, en español e inglés

Referencia del autor o autores:

Institución de adscripción, grado académico y líneas-grupos de investigación que desarrolla y a los que pertenece.

\section{Sistema de referencia de citas:}

Harvard-APA

Las citas bibliográficas en el texto deberán incluir entre paréntesis sólo el apellido del autor, la fecha de publicación y el número de página; por ejemplo: (Quilodrán, 2001: 33).

\section{Notación en sección de bibliografía y fuentes de información:}

Se deberá incluir al final del texto. Toda referencia deberá estar mencionada en el texto o notas de pie de página.

Cada referencia iniciará con el primer apellido o los apellidos, luego el nombre del autor, y después, entre paréntesis, el año de publicación seguido de un punto. Ejemplos:

Se deberá incluir al final del texto. Toda referencia deberá estar mencionada en el texto o notas de pie de página.

Cada referencia iniciará con el primer apellido o los apellidos, luego el nombre del autor, y después, entre paréntesis, el año de publicación seguido de un punto. Ejemplos:

Artículo:

Ros, Jaime (2008). "La desaceleración del crecimiento económico en México desde 1982”, en Trimestre Económico, vol. 75, núm. 299, pp. 537-560.

Libro:

Villarreal, René (2005). Industrialización, competitividad y desequilibrio externo en México. 
Un enfoque macroindustrial y financiero (1929-2010), México, Fondo de Cultura Económica. Capítulo de libro:

Castillo, Manuel Ángel (2003). "La política de inmigración en México: un breve recuento", en Manuel Ángel Castillo, Alfredo Lattes y Jorge Santibáñez (coords.), Migración y fronteras, Tijuana, El Colegio de la Frontera Norte / Asociación Latinoamericana de Sociología / El Colegio de México, pp. 425-451.

\section{Notas de pie de página:}

Se utilizarán para hacer indicaciones complementarias, aclaraciones o ampliación de una explicación. La notas de pie de página en Times New Roman, 10 puntos.

\section{Tipología de imágenes dentro del texto:}

Cuadro

Gráfica

Diagrama

Mapa

Figura

Todas las imágenes deben ser numeradas y mencionadas dentro del texto. A toda imagen debe incluirse la fuente.

Las indicaciones de la imagen: tipo y número de imagen, título de imagen y fuente se escriben en 10 puntos. En el texto poner como imagen los mapas, figuras, gráficas y diagramas -con el ánimo de no perder el formato realizado por el autor.

\section{Ecuaciones y fórmulas:}

Si se utilizan ecuaciones o fórmulas deberá utilizarse el editor de ecuaciones de Word y numerarse.

\section{Envío de trabajos}

Los trabajos deben ser enviados a la dirección de correo: lgtz@uacj.mx. Con el Dr. Luis Enrique Gutierrez Casas, editor de esta publicación.

La aceptación de cada colaboración dependerá de la evaluación de dos dictaminadores especialistas en la materia que se conservarán en el anonimato, al igual que el autor (autores) para efectos de la misma. 


\section{$\rightarrow$ Editorial Guidelines}

\section{For General Document:}

Font type: Times New Roman.

Size: font size 11 .

Paragraph: 1.5 line spacing.

Titles and subtitles: Main text font size 11. Titles font size 12 (Bold). Subtitles font size 11.

Each title and subtitle should be numbered in the following order: 1, 1.1, 2, 2.1, 2.2...

The maximum length of the workbooks will be 40 pages.

The first time an abbreviation is used in the text will be specified first complete equivalence and then stands.

\section{Front cover:}

Title:

Font size 14, centered, Bold.

Author name(s):

Font size 12.

Abstract:

It should include abstract in Spanish and abstract (font size 10), no more than 250 words.

Keywords:

Include three to five keywords, in Spanish and English.

Reference of author:

Institution of affiliation, academic degree and line-developed by research groups and belonging.

\section{Bibliographical appointment system:}

Harvard-APA

Citations in the text should include between parentheses only the author's name, publication date and page number, for example:

(Quilodrán, 2001: 33).

\section{Notation about Bibliography section and Information fonts:}

Should be included at the end of the text. All references must be mentioned in the text or footnotes page.

Each reference starts with the first name or last name, then the name of the author, and then, in parentheses, the year of publication followed by a period. Examples:

Article:

Ros, Jaime (2008). “La desaceleración del crecimiento económico en México desde 1982”, en Trimestre Económico, vol. 75, núm. 299, pp. 537-560.

Book:

Villarreal, René (2005). Industrialización, competitividad y desequilibrio externo en México. Un enfoque macroindustrial y financiero (1929-2010), México, Fondo de Cultura Económica.

Book chapter:

Castillo, Manuel Ángel (2003). "La política de inmigración en México: un breve recuento”, en Manuel Ángel Castillo, Alfredo Lattes y Jorge Santibáñez (coords.), Migración y fronteras, Tijuana, E1 Colegio de la Frontera Norte / Asociación Latinoamericana de Sociología / El Colegio de México, pp. 425-451. 


\section{Footnotes:}

Must be used to make additional indications, clarification or expansion of an explanation. The footnotes must be in Times New Roman, font size 10.

\section{Image typology inside text:}

Picture

Graph

Diagram

Map

Figure

All images must be numbered and mentioned in the text, should include the source image. The indications of the image: type and number of image, image title and source are written in 10 font size. In the text set as image maps, figures, graphs and charts-with the intention of not losing the formatting by the author.

\section{Equations and Formulae:}

When using equations or formulas should be used in Microsoft Word equation editor and numbered.

\section{Paper sending}

Entries must be sent to the email address: lgtz@uacj.mx. With Dr. Luis Enrique Gutiérrez Casas, editor of this publication.

Acceptance of each collaboration will depend on the evaluation of two examiners skilled in the art to be kept anonymous, like the author(s) for the same purposes. 


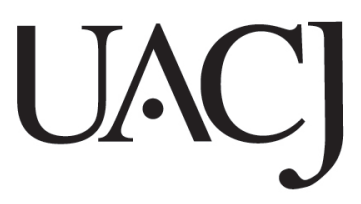

Esta obra se terminó de imprimir en junio de 2018 Cd. Juárez, Chihuahua, México.

Tiraje: 120 ejemplares 
Cuadernos de Trabajo de la Universidad Autónoma de Ciudad Juárez, número 46, julio - agosto de 2018

\section{Director y editor}

Dr. Luis Enrique Gutiérrez Casas

\section{Comité editorial}

Sección internacional

Dra. Sofía Boza Martínez (Universidad de Chile, Chile)

Dra. Olga Biosca Artiñano (Glasgow Caledonian University, Reino Unido)

Dra. Ángeles Sánchez Díez (Universidad Autónoma de Madrid, España)

Dr. Thomas Fullerton Mankin (University of Texas at El Paso, Estados Unidos)

Dr. Adrián Rodríguez Miranda (Universidad de la República, Uruguay)

\section{Sección local}

(Universidad Autónoma de Ciudad Juárez)

Dra. Myrna Limas Hernández

Dra. Ikuho Kochi

Dr. Raúl Alberto Ponce Rodríguez

Dr. Isaac Leobardo Sánchez Juárez

Dr. Héctor Alonso Barajas Bustillos 